CERN-EP/2003-026

15 May 2003

\title{
Space Charge Effects in Resistive Plate Chambers
}

\author{
Christian Lippmann ${ }^{1)}$ and Werner Riegler ${ }^{1)}$
}

\begin{abstract}
We extend an existing one dimensional simulation model for avalanche mode RPCs by the inclusion of diffusion and space charge effects. We investigate the influence of space charge on charge spectra, signal rise time, time resolution and charge-to-time correlation. We present a 1.5-dimensional model, which allows fast simulation of events and a two dimensional model that is slower but allows to study the transverse dispersion of avalanches due to space charge.
\end{abstract}

Preprint submitted to Elsevier Science

1) EP Division, CERN, CH-1211 Geneva 23, Switzerland 
Introduction

Resistive Plate Chambers (RPCs) [1,2] operated in avalanche mode at atmospheric pressure offer an affordable and reliable technology for detectors for present and future high energy physics experiments. Large area RPCs with a $2 \mathrm{~mm}$ single gas gap (Trigger RPCs) operated in avalanche mode provide above $98 \%$ efficiency and a time resolution of around $1 \mathrm{~ns}$ up to a flux of several $\mathrm{kHz} / \mathrm{cm}^{2}[3,4]$. They are constructed of two resistive electrode plates made of bakelite as shown schematically in Fig. 1a. Timing RPCs [5] with gas gaps of 0.2 to $0.3 \mathrm{~mm}$ are used in multi gap configurations [6] for Time-Of-Flight purposes [7]. They provide $99 \%$ efficiency and time resolutions down to $50 \mathrm{ps}[8,9,10,11]$. Different technologies with one or more resistive electrode plates are used. The two single gap designs investigated in this publication are shown in Fig. 1.

a)

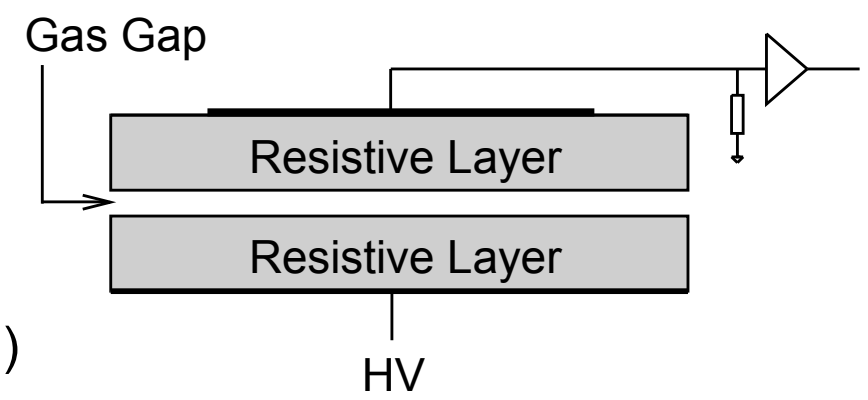

b)

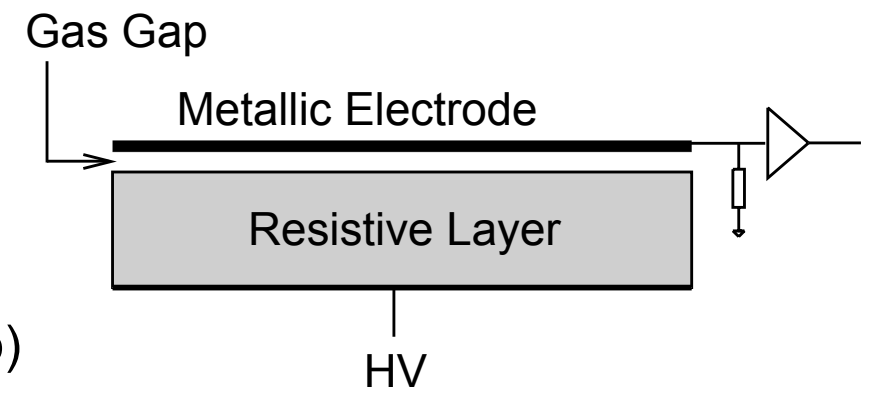

Figure 1: a) Single gap RPC with two resistive electrode plates. b) Single gap RPC with one resistive electrode plate as in $[8,9,10]$.

The Monte-Carlo simulation of the physical processes in particle detectors is an important tool for understanding the behavior of the detectors in the particle physics experiments in which they are or will be implemented. In order to optimize the detector physics parameters like gas mixture, gas pressure, gas gain and electronics parameters like preamplifier peaking time, noise, threshold settings etc., generally a detailed simulation of the detector response is carried out. For the simulation of wire and drift chambers one often utilizes the simulation tool GARFIELD [12]. For the simulation of RPCs no such tool exists. As a consequence, many experimental results have not been properly studied. Even though the geometry of the device is much simpler than that of a wire based detector, there are still disagreements about the explanation of several aspects of the performance of RPCs [13]. Thus the need for a detailed Monte-Carlo simulation of avalanches in RPCs arose. In [14] we presented a Monte-Carlo model suitable to simulate the time resolution and efficiencies of RPCs. It turns out that neglecting a space charge effect (see Fig. 2), the simulated average charges and shapes of the charge spectra differ largely from measurements. A saturation due to the space charge effect can be simulated in a crude way 


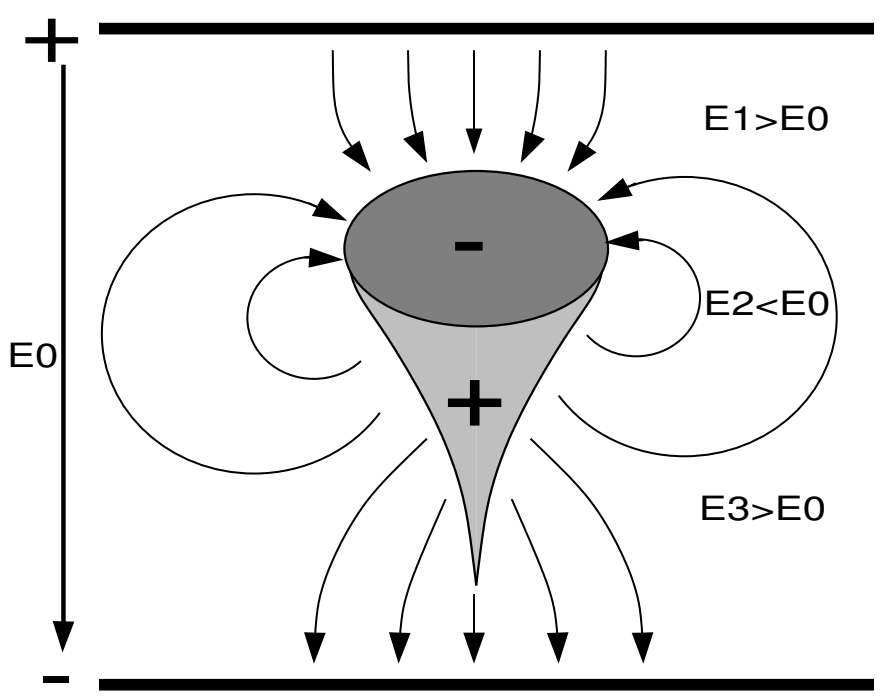

Figure 2: A schematic picture of an avalanche and the electric field deformations caused by the avalanche charge carriers. At the tip and the tail of the charge distribution the fields $E_{1}$ and $E_{3}$ are higher than the applied electric field $E_{0}$. In the center of the charge distribution the field $E_{2}$ is lower than $E_{0}$. As a consequence, the values of the gas parameters like drift velocity and Townsend coefficient are not uniform in the gas gap in the gas gap.

by cutting off the avalanche growth at a certain size [15]. A different approach is proposed in $[16,17]$, where the saturated growth is explained by a constant-coefficient, non-linear differential equation that is also used to describe the evolution of a biological population in a limited resources environment. In [18] a space charge effect is included by introducing a functional dependence of the effective Townsend coefficient $\alpha_{e f f}$, that describes the average avalanche multiplication $\left(n(z)=e^{\alpha_{e f f} z}\right)$, on the avalanche size.

A much more accurate approach involves the dynamic calculation of the electric field contributed by the avalanche charges. We follow this approach and describe the detector physics of the RPC using only well-defined fundamental physics parameters. For the values of the gas parameters and their dependence on the electric field we use the predictions by the simulation programs MAGBOLTZ [23] and IMONTE [24] (see Figs. 3 and 4). For the primary ionization parameters (mean free path and cluster size distribution) we use the program HEED [25] (for plots, see [14]). Extending the basic model from [14], we use analytic formulas for the potential of a point charge in a three layer geometry like the RPC [19, 20] (Fig. 1a). With the calculated values for the electric field of the space charge we further calculate dynamically the actual values of the gas parameters like drift velocity and Townsend coefficient. This approach ensures an understanding and description of the evolution of avalanches in RPCs in a very elementary way. Some first results obtained with this model (the so-called "1.5-D model", see section 2) were presented in $[21,22]$. In this publication we present the progress made in the simulation of electron avalanches using the 1.5-D model. Moreover we introduce and describe a two dimensional avalanche simulation Model (2-D model) and present results obtained with it. 

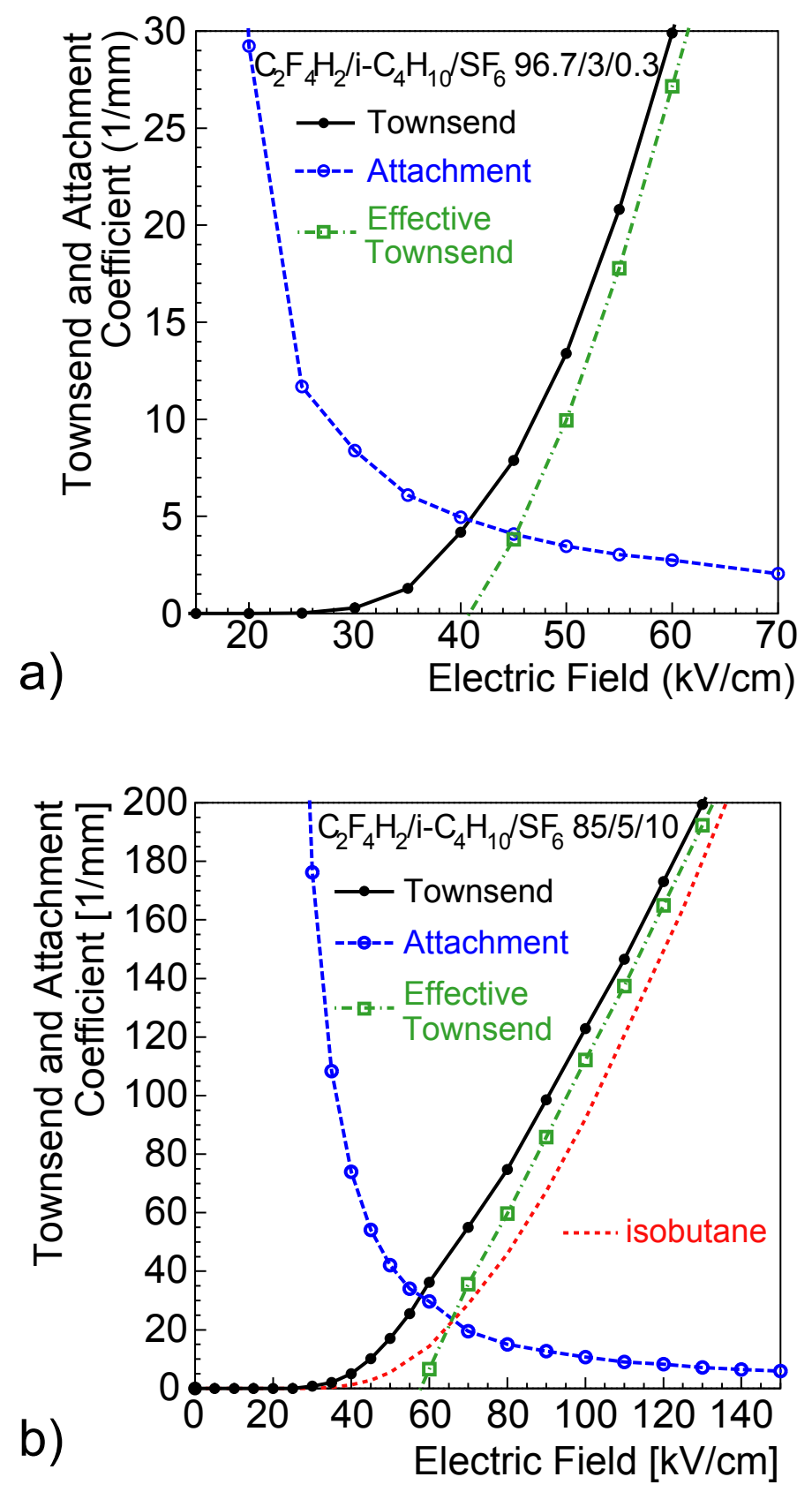

Figure 3: Townsend and attachment coefficients for different gas mixtures calculated with IMONTE at $T=296.15 \mathrm{~K}$ and $p=1013$ mbar [14]. a) A typical Trigger RPC gas mixture. b) A typical Timing RPC gas mixture and pure isobutane. 

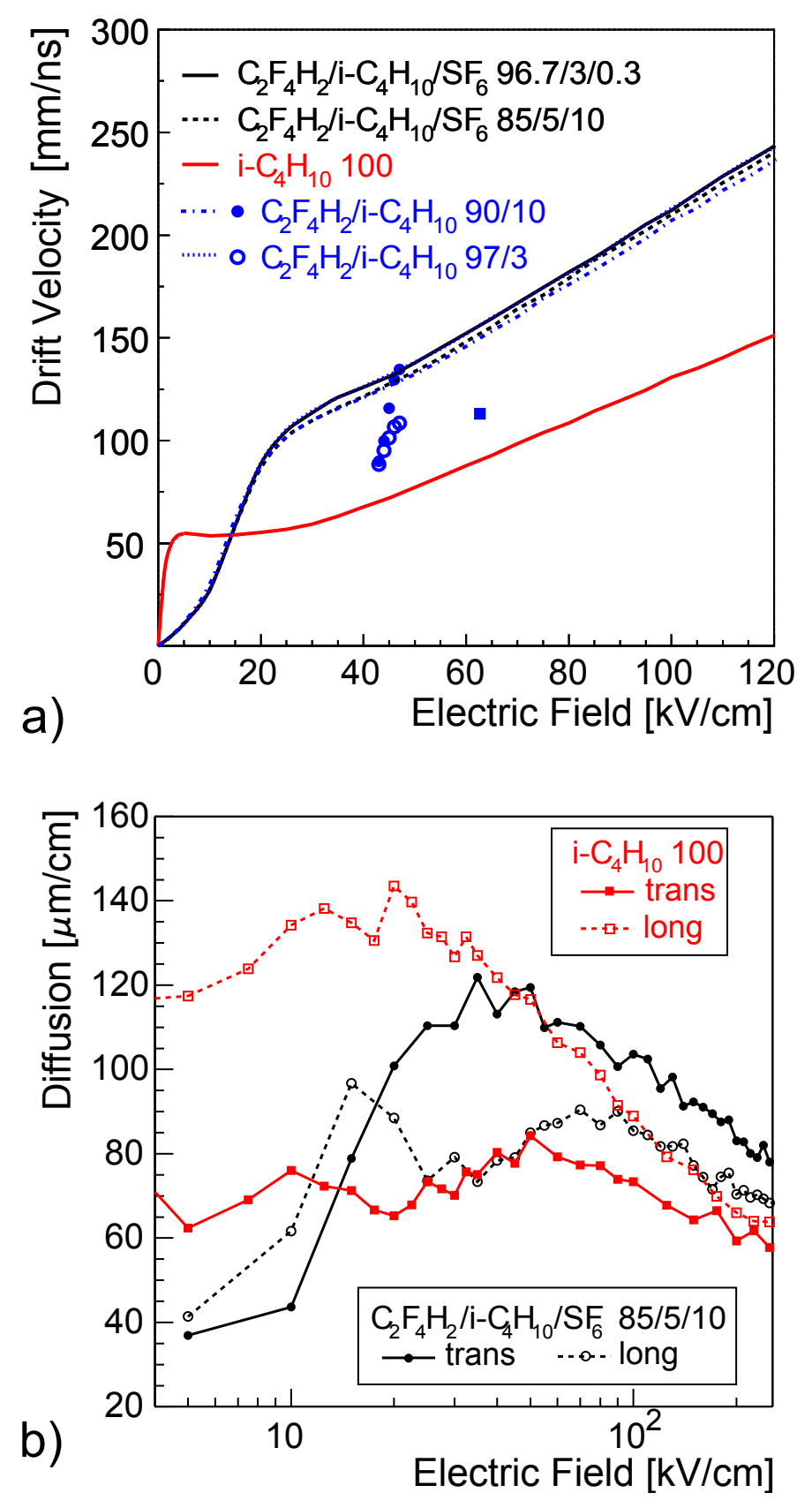

Figure 4: a) Drift velocities calculated with MAGBOLTZ for different gas mixtures together with measurements. The circles show measurements from [26] for two different mixtures and the square shows a measurement from [27] for $\mathrm{C}_{2} \mathrm{~F}_{4} \mathrm{H}_{2} / \mathrm{i}-\mathrm{C}_{4} \mathrm{H}_{10} / \mathrm{SF}_{6}(96.9 \%, 3 \%, 0.1 \%)$. b) Diffusion coefficients calculated with MAGBOLTZ for $\mathrm{C}_{2} \mathrm{~F}_{4} \mathrm{H}_{2} / \mathrm{i}-\mathrm{C}_{4} \mathrm{H}_{10} / \mathrm{SF}_{6}(85 \%, 5 \%, 10 \%)$, which is often used in Timing RPCs, and for isobutane. The data for the gas mixture $\mathrm{C}_{2} \mathrm{~F}_{4} \mathrm{H}_{2} /$ $\mathrm{i}-\mathrm{C}_{4} \mathrm{H}_{10} / \mathrm{SF}_{6}(96.7 \%, 3 \%, 0.3 \%)$ is very similar to the one with $10 \% \mathrm{SF}_{6}$ and is not shown. All values are for $T=296.15 \mathrm{~K}$ and $p=1013 \mathrm{mbar}$. 


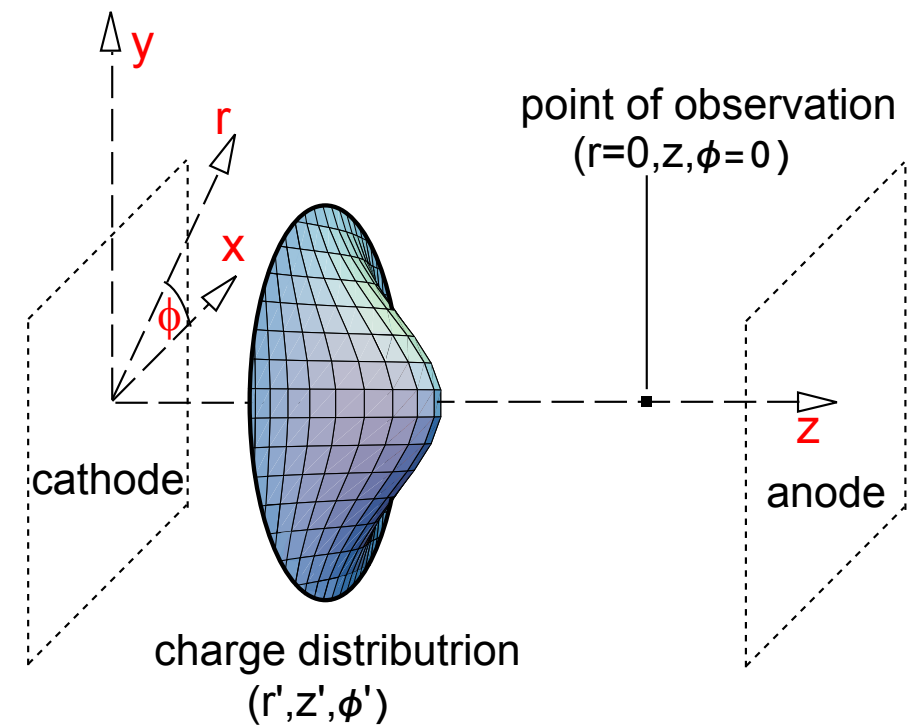

Figure 5: The geometry for the 1.5-D simulation. The point of observation is $(r, \phi, z)$ and the disk with the Gaussian radial charge distribution is positioned at $z^{\prime}$. Since the simulation is performed only along the $z$-axis, it is sufficient to calculate the field only at $(r=0, \phi=0, z)$.

\section{$2 \quad$ The 1.5-D Model}

We extend the 1-D avalanche simulation model described in [14] by including diffusion and space charge effects. The basic structure of the simulation is the following:

1. The $z$-axis (see Fig. 5) is crossing the gas gap perpendicular to the electrode plates. It is divided into $N$ steps of size $\delta z=g / N$ corresponding to time steps of $\delta t=\delta z / v_{0}$, where $g$ is the gap size and $v_{0}=v_{D}\left(E_{0} / p\right)$ is the electron drift velocity at the applied electric field $E_{0}$ and at pressure $p$.

2. We assume that all particle tracks are perpendicular to the electrode plates of the detector.

3. The primary clusters are distributed onto the steps, with distances following an exponential distribution with a mean corresponding to the mean free path for primary ionization.

4. Primary electrons are put to each cluster, following the cluster size distribution.

5. The electric field $E(z)$ at all steps where electrons are situated is calculated. Here we also include transverse diffusion. The procedure is described in detail in section 2.1.

6. The drift velocity $v_{D}(E(z) / p)$, the Townsend coefficient $\alpha(E(z) / p)$ and the attachment coefficient $\eta(E(z) / p)$ are calculated at each step where electrons are found (Figs. 3 and 4).

7. The avalanches for each single electron are simulated using the procedure outlined in [14]. We also include longitudinal diffusion by drawing a random number from a Gaussian distribution with a standard deviation depending on the longitudinal diffusion coefficient $D_{L}\left(E_{0} / p\right)[\sqrt{\mathrm{cm}}]$. We use the constant value of $D_{L}$ at field $E_{0}$. The charges are redistributed onto the steps. A resistive anode plate is simulated by letting the electrons that reach this plate 'stick' to its surface.

8. At each time step, the current and charge induced by the drifting electrons are calculated with the help of the weighting field formalism and the Ramo theorem [28].

9. Steps 5 to 8 are repeated until all electrons have left the gas gap. 

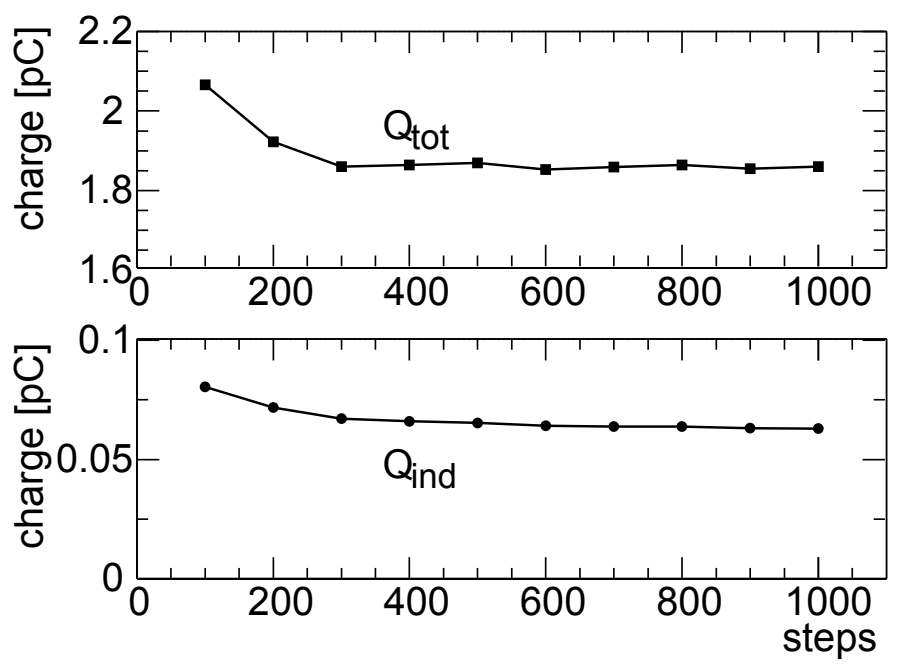

Figure 6: The average signal charge $Q_{t o t}$, which is the charge of the positive ions in the gas gap at the end of the avalanche development, and the induced charge $Q_{\text {ind }}$ for different numbers of steps. We used a $0.3 \mathrm{~mm}$ gap Timing RPC at $2.7 \mathrm{kV}$ and simulated average avalanches. For a number of steps larger than 200 we find a fluctuation of $2.2 \%$ and $0.3 \%$ r.m.s. for $Q_{i n d}$ and $Q_{t o t}$.

\subsection{Calculation of the Space Charge Field}

We use cylindrical coordinates $r, z$ and $\phi$ and assume that the avalanche has rotational symmetry around the $z$-axis. The longitudinal avalanche propagation is completely simulated, while the transverse spread is included by considering the charges at each step to be contained in disks with a Gaussian charge distribution in the radial direction. This explains the terminology "1.5-D" used to refer to this model. The standard deviation of the Gaussian depends on the transverse Diffusion coefficient $D_{T}\left(E_{0} / p\right)[\sqrt{\mathrm{cm}}]$ and on the drifted distance. We use the constant value of $D_{T}$ at field $E_{0}$. The $z$-component of the electric field of a disk at position $z^{\prime}$ containing the unit charge is calculated as the space integral over the mentioned radial charge distribution and the potential of a point charge in an infinite plane condenser comprising three homogeneous layers. An analytic solution for this potential is given in [19, 20]. The positions of the charge distribution and the point of observation are shown schematically in Fig. 5. The field of all the charge in the gap (the field of the space charge) is calculated by integration (summation) over all the disks.

We can now calculate the field of the space charge in the gas gap at all positions. Above a certain step number the calculation is only very slightly depending on the chosen step size, which is shown in Fig. 6.

\subsection{Results for Trigger RPCs}

The average charges in $2 \mathrm{~mm}$ gap Trigger RPCs obtained with the Monte-Carlo simulation without a space charge effect but with longitudinal diffusion are:

$$
Q_{\text {ind }} \approx 7.9 \times 10^{3} \mathrm{pC}, \quad Q_{\text {tot }} \approx 2.6 \times 10^{5} \mathrm{pC} .
$$

In experiment one measures values that are several orders of magnitudes smaller [29]:

$$
Q_{\text {ind }} \approx 2 \mathrm{pC}, \quad Q_{\text {tot }} \approx 40 \mathrm{pC}
$$



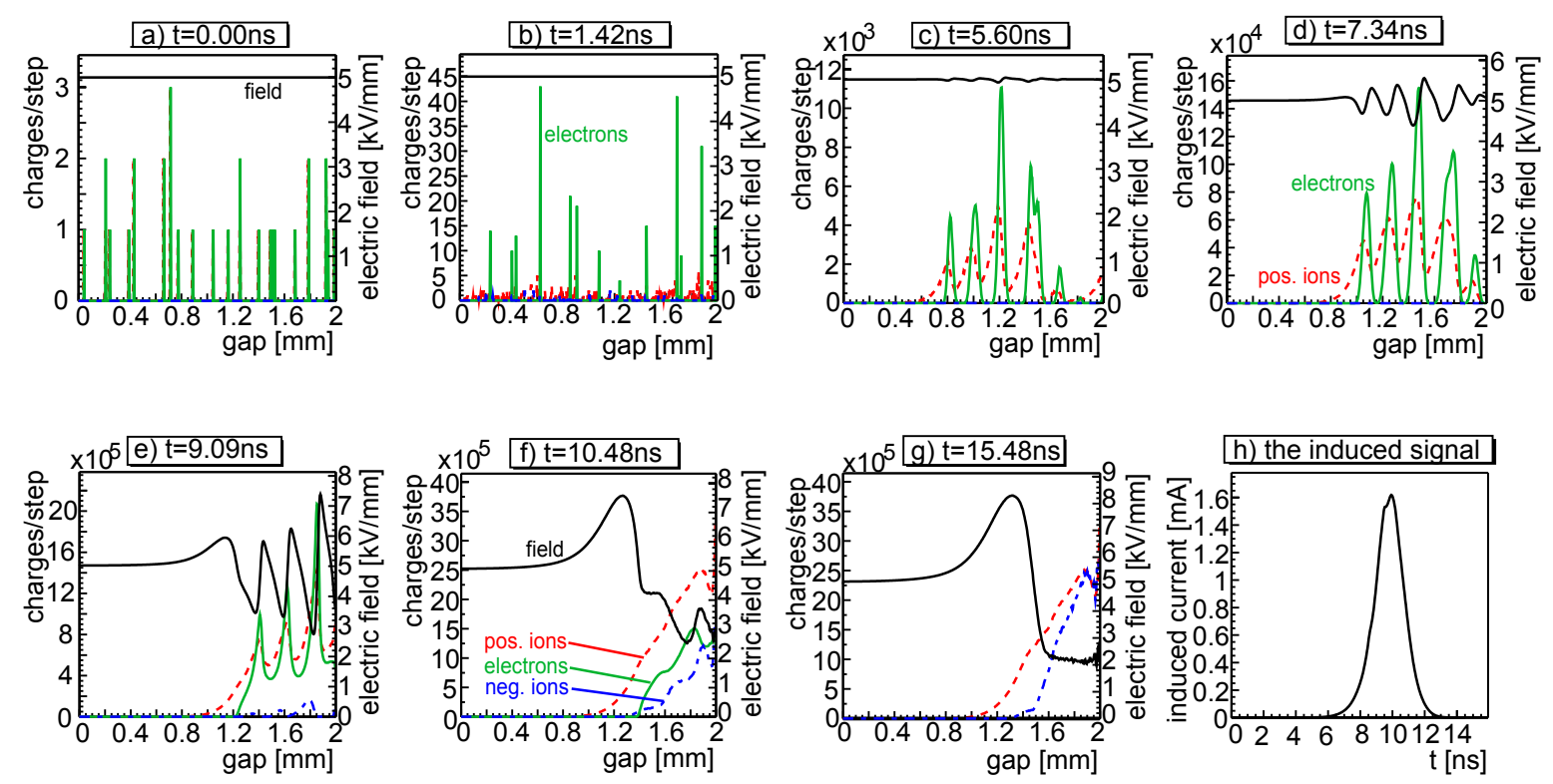

Figure 7: A simulated avalanche in a Trigger RPC. We show snapshots of the charge configuration in a $2 \mathrm{~mm}$ gas gap (500 steps). The distributions of electrons and positive and negative ions are shown and correspond to the axes on the left. The $z$-components of the electric field across the gap is also plotted and correspond to the axes on the right. The last image is the induced current signal.

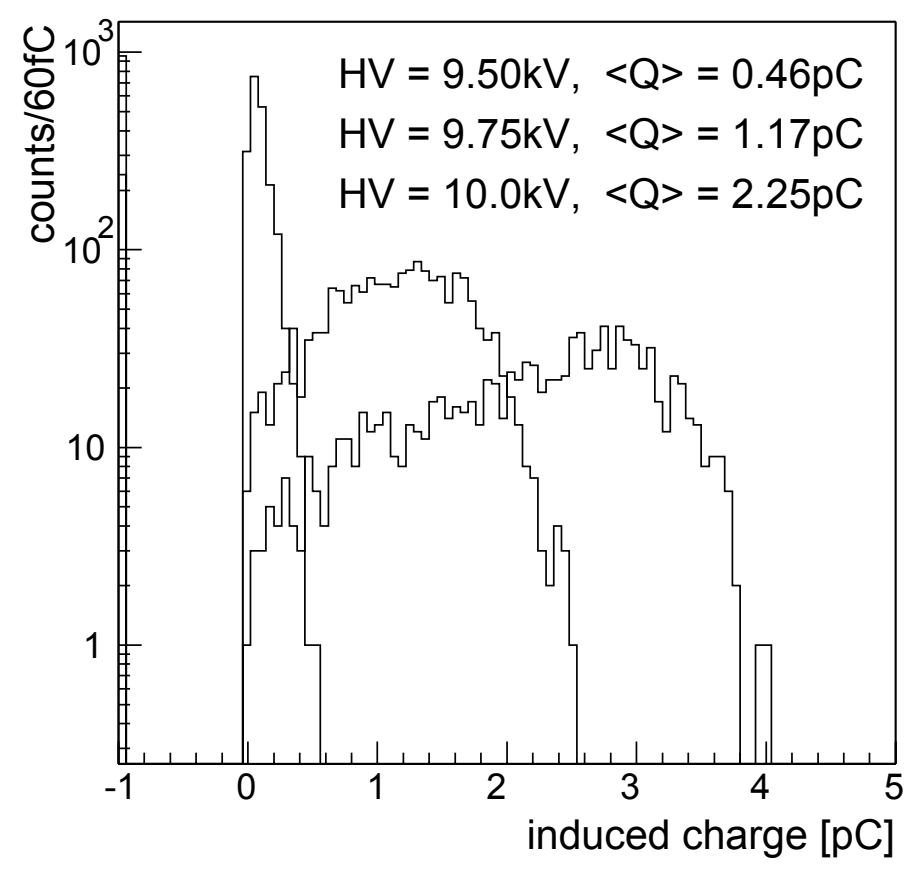

Figure 8: Simulated induced charge spectra for single gap Trigger RPCs filled with $\mathrm{C}_{2} \mathrm{~F}_{4} \mathrm{H}_{2}$ / i- $\mathrm{C}_{4} \mathrm{H}_{10} / \mathrm{SF}_{6}(96.7 \%, 3 \%, 0.3 \%)$. The average values take into account only charges larger than a threshold of $100 \mathrm{fC}$. The entries at -1 correspond to the unstable events (only at $10 \mathrm{kV}$ ). The temperature is $T=296.15 \mathrm{~K}$ and the pressure $p=970 \mathrm{mbar}$. 
We observe a discrepancy of up to 4 orders of magnitude! The measured small avalanche charges are explained by a space charge effect. A typical simulated avalanche in a single gap Trigger RPC filled with $\mathrm{C}_{2} \mathrm{~F}_{4} \mathrm{H}_{2} /$ i- $\mathrm{C}_{4} \mathrm{H}_{10} / \mathrm{SF}_{6}(96.7 \%, 3 \%, 0.3 \%)$ at $10 \mathrm{kV}$ is shown in Fig. 7:

(a) At $t=0 \mathrm{~ns}$ the passage of a $120 \mathrm{GeV}$ muon generates 20 primary clusters with between one and three electron-ion pairs each. The electrons are then drifting towards the anode at $z=2 \mathrm{~mm}$.

(b) Some of the primary electrons get attached or enter the anode resistive layer at $t=$ $1.42 \mathrm{~ns}$.

(c) The space charge begins to influence the electric field at $t=5.6 \mathrm{~ns}$ (compare to Fig. 2).

(d) At $t=7.34 \mathrm{~ns}$ the space charge field reaches around $10 \%$ of the applied electric field strength.

(d) At $t=9.09 \mathrm{~ns}$ the space charge field reaches around $40 \%$ of the applied electric field strength.

(e) At $t=10.48 \mathrm{~ns}$ the field drops dramatically in a large fraction of the gas gap due to the large amount of negative charge (electrons) that has entered the resistive anode. Due to the high resistivity of the material this charge is sticking to the anode surface for a time that is much longer than the signal time ${ }^{1)}$. From now on the field is very low in all areas where electrons are situated. As a consequence we find strong attachment and low values of the electron drift velocity (see Figs. 3a and 4a).

(f) At $t=15.48 \mathrm{~ns}$ all electrons have either left the gap or got attached. The field in front of the anode is lowered to only $40 \%$ of the applied electric field.

(h) In the last image we show the induced current signal.

We accumulated spectra of the induced charge including a space charge effect in the simulation for single gap Trigger RPCs at different high voltages $(9.5 \mathrm{kV}, 9.75 \mathrm{kV}$ and $10 \mathrm{kV})$. We chose 500 steps for the 1.5-D simulation procedure. In Fig. 8 we show the induced charge spectra. At $10 \mathrm{kV}$ and higher voltages, the simulation tends to be unstable and some events show an exploding electric field. If this behavior is detected the simulation of the current event is stopped and skipped. The charges of these events are missing in the spectra and thus in the calculation of the average charges. Since the skipped events are generally events that would have given rather large charges, the average induced charge at $10 \mathrm{kV}\left(\left\langle Q_{\text {ind }}\right\rangle=2.25 \mathrm{pC}\right)$ and also the average total signal charge $\left(\left\langle Q_{t o t}\right\rangle=33.6 \mathrm{pC}\right)$ might be somewhat too small. Nevertheless, we find numbers for the average charges that are very similar to measurements. Also the shapes of the spectra are very similar to measured data (for example, see [29]).

\subsection{Results for Timing RPCs}

\subsubsection{Charge Spectra}

The average charges obtained with the Monte-Carlo simulation for $0.3 \mathrm{~mm}$ single gap Timing RPCs without a space charge effect but with longitudinal diffusion are:

$$
Q_{\text {ind }} \approx 5 \times 10^{7} \mathrm{pC}, \quad Q_{\text {tot }} \approx 5 \times 10^{9} \mathrm{pC},
$$

In the experiment one measures values that are several orders of magnitudes smaller $[8,9,10]$ :

$$
Q_{\text {ind }} \approx 0.3 \mathrm{pC}, \quad Q_{\text {tot }} \approx 5 \mathrm{pC}
$$



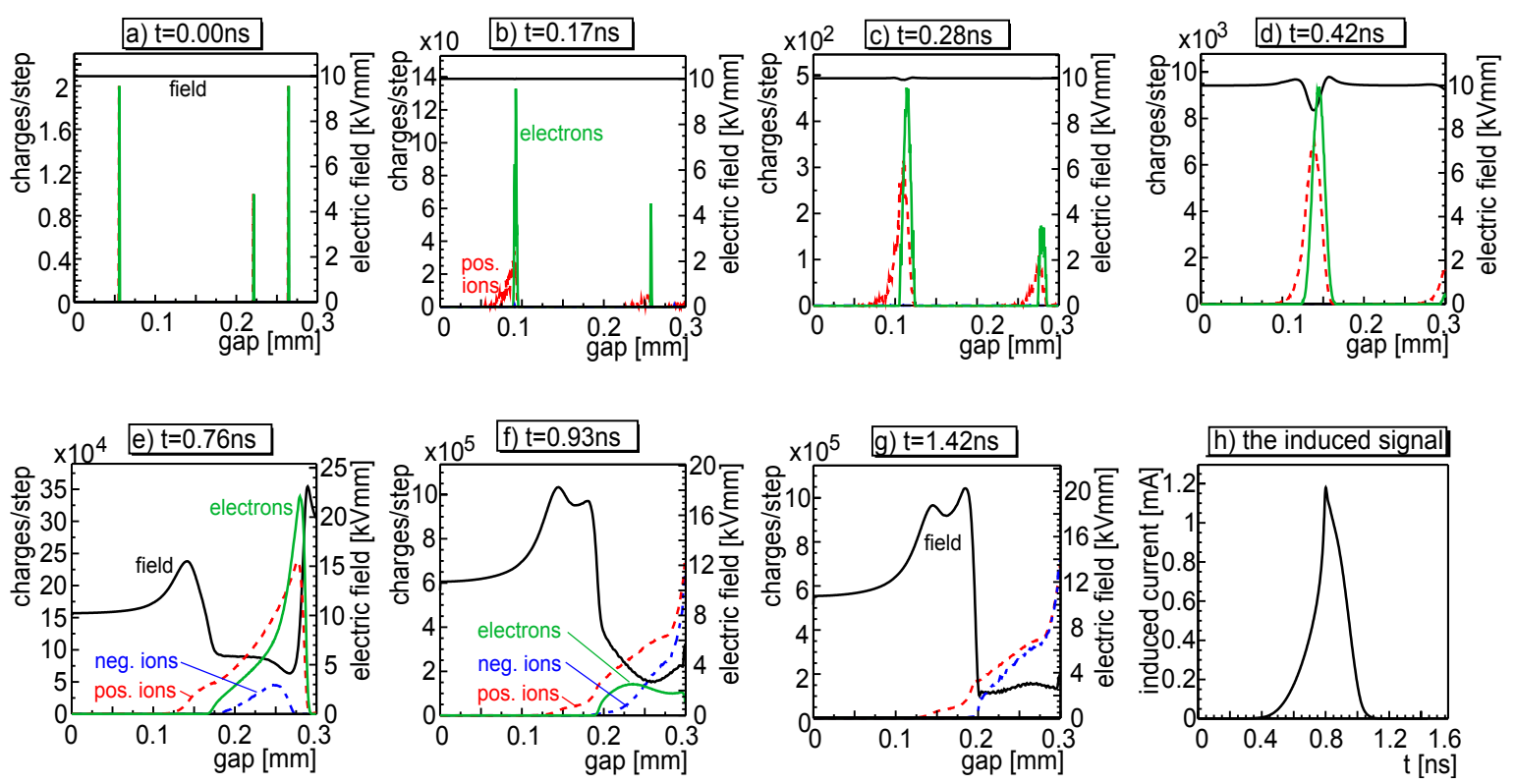

Figure 9: A simulated avalanche in a Timing RPC. We show snapshots of the charge configuration in a $0.3 \mathrm{~mm}$ gas gap (500 steps). The distributions of electrons, positive and negative ions are shown and correspond to the axes on the left. The $z$-components of the electric field across the gap is also plotted and correspond to the axes on the right. The last image is the induced current signal.

We observe a discrepancy of up to nine orders of magnitude! We chose 500 steps for the 1.5-D simulation of avalanches and accumulated charge spectra of the induced charge $Q_{\text {ind }}$ and of the total signal charge $Q_{t o t}$ for Timing RPCs including a space charge effect. A typical simulated avalanche in a $0.3 \mathrm{~mm}$ single gap Timing RPC filled with $\mathrm{C}_{2} \mathrm{~F}_{4} \mathrm{H}_{2} / \mathrm{i}-\mathrm{C}_{4} \mathrm{H}_{10} / \mathrm{SF}_{6}$ $(85 \%, 5 \%, 10 \%)$ at a high voltage of $3 \mathrm{kV}$ is shown in Fig. 9:

(a) In the first image at $t=0 \mathrm{~ns}$ the passage of a $7 \mathrm{GeV}$ pion generates three primary clusters; one cluster consisting of one electron-ion pair and two clusters with two electron-ion pairs each. The electrons are then drifting towards the anode at $z=0.3 \mathrm{~mm}$.

(b) The electrons in the cluster closest to the anode have left the gas gap at $t=0.17 \mathrm{~ns}$.

(c) The two remaining electron clusters grow further.

(d) The second electron cluster has left the gap at $t=0.42 \mathrm{~ns}$. We observe how the space charge begins to influence the electric field. The field is increased at the tip and at the tail of the last electron cloud in the gas gap while at its center it is about $15 \%$ lower. As a consequence, we find regions with increased multiplication and regions with slower multiplication. Because of the resistivity of the anode layer all charges that reach the anode 'stick' to the electrode surface. Their presence influences the electric field in front of the anode.

(e) The closer the cluster approaches the anode, the higher the field at its tip gets. At $t=$ $0.76 \mathrm{~ns}$ the maximum space charge field has a value that exceeds the applied electric field (at the tip of the avalanche) while in the center of the electron cloud the field is halved. Here we find strong attachment of electrons and large amounts of negative ions are formed.

1) The time constant $\tau$ is given by $\tau=\rho \varepsilon_{0} \varepsilon_{r}$, where $\rho[\Omega \mathrm{cm}]$ is the volume resistivity of the material, $\varepsilon_{0}$ is the dielectric constant of vacuum and $\varepsilon_{r}$ is the relative permittivity of the resistive material 
(f) Due to the many electrons that have entered the resistive anode surface the field drops dramatically. From now on there is strong attachment of the remaining electrons in the gas gap and the drift velocity is decreased. The amount of negative ions in front of the anode grows rapidly.

(g) At $t=1.42 \mathrm{~ns}$ all electrons have either left the gap or got attached. The field in front of the anode is lowered to only $40 \%$ of the applied electric field.

(h) In the last image we show the induced current signal.

In Fig. 10 we show simulated and measured spectra of the total signal charge in a $0.3 \mathrm{~mm}$ single gap Timing RPC at high voltages of $2.3 \mathrm{kV}, 2.5 \mathrm{kV}$ and $2.8 \mathrm{kV}$. The mean of the simulated spectra is around a factor two larger than the measured one. But as compared with the discrepancy of several orders of magnitude that we observed if a space charge is neglected, we can consider the simulation to be close to the measured values. Also the shape of the spectra is very similar to the measured data.

An uncertainty concerning the value of the Townsend and attachment coefficients $\alpha(E / p)$ and $\eta(E / p)$ at large electric field strengths is a possible reason for the small deviation of the average values of the spectra. It turns out that a decrease of the value of $\alpha(E / p)$ by $10 \%$ leads to a decrease of the mean value of the spectra by 30\%. Since measurements of $\alpha(E / p)$ and $\eta(E / p)$ at the large values of the electric fields observed in RPCs are not available, an error of $10 \%$ or even more is certainly imaginable.

\subsubsection{Operational Mode of RPCs}

From wire chambers filled with a quench gas with good UV absorption it is known that for certain high voltages one observes a region where the charge is proportional to the primary charge (proportional mode). Here the charge increases exponentially with the high voltage. After this one encounters the very narrow space charge mode of usually less than one hundred Volts where the charge growth deviates from the exponential. When further increasing the high voltage, the average charge suddenly increases by a factor 10 to 100 (limited streamer mode). At even higher voltages, the charge continues to rise more slowly up to the general breakdown of the chamber or the Geiger-Müller mode.

For parallel plate geometries like RPCs and neglecting space charge effects we expect an exponential dependence of the charge on the effective Townsend coefficient $\alpha_{\text {eff }}=\alpha-\eta$. Since at high fields the dependence of $\alpha_{e f f}$ on the field $E$ is approximately linear (Fig. 3), the relation between the charge and $E$ will be approximately exponential, as for wire chambers. As can be seen in Fig. 11, the Timing RPC shows this exponential behavior at low fields, which is however giving charges that are too small for efficient detection. We also observe that in the broad operational region (in the case of the $0.3 \mathrm{~mm}$ gap Timing RPC from around 9 to $11 \mathrm{kV} / \mathrm{mm}$ ) the detector is operated in space charge mode.

The value of the charge depends first exponentially on the applied high voltage but then the dependence becomes approximately linear, which is also an observed experimental fact (for example, see Fig. 3 in [30] and Fig. 5 in [9]). Only at very high fields the occurrence of streamers is experimentally observed, which limits the space charge region towards higher voltages.

\subsubsection{Signal Rise Time}

At the discriminator threshold level of 10 to $100 \mathrm{fC}$ the avalanche in an RPC generally consists of more than $10^{6}$ electrons. It turns out that at that level the space charge effect does already have an influence on the avalanche growth and hence the signal rise time. Fig. $12 \mathrm{~b}$ shows the intrinsic time resolutions for single gap Timing RPCs at $3 \mathrm{kV}$ for two cases: In the first case we included the space charge effect in the simulation, in the second we did not include 


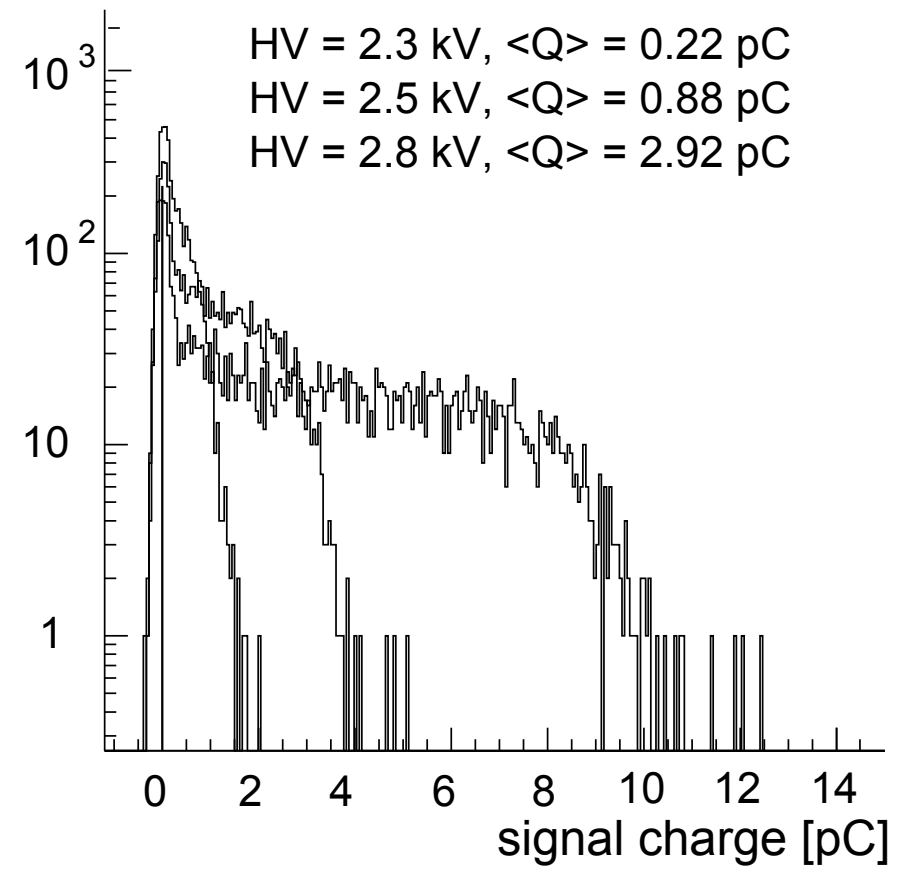

a) Simulation

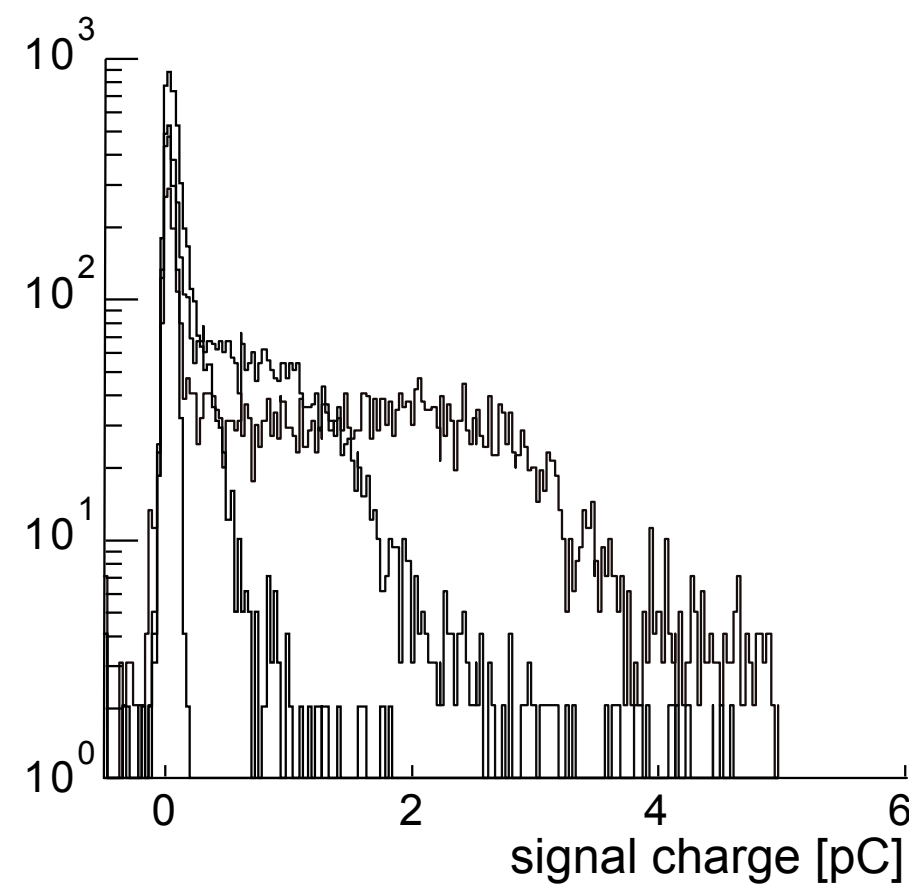

b) Measurement

Figure 10: a) Simulated total signal charge spectra for $0.3 \mathrm{~mm}$ single gap Timing RPCs. The mean values take into account all data including the inefficient events. $100 \mathrm{fC}$ noise is added. The temperature is $T=296.15 \mathrm{~K}$ and the pressure $p=970$ mbar. b) Measured spectra from [9]. 

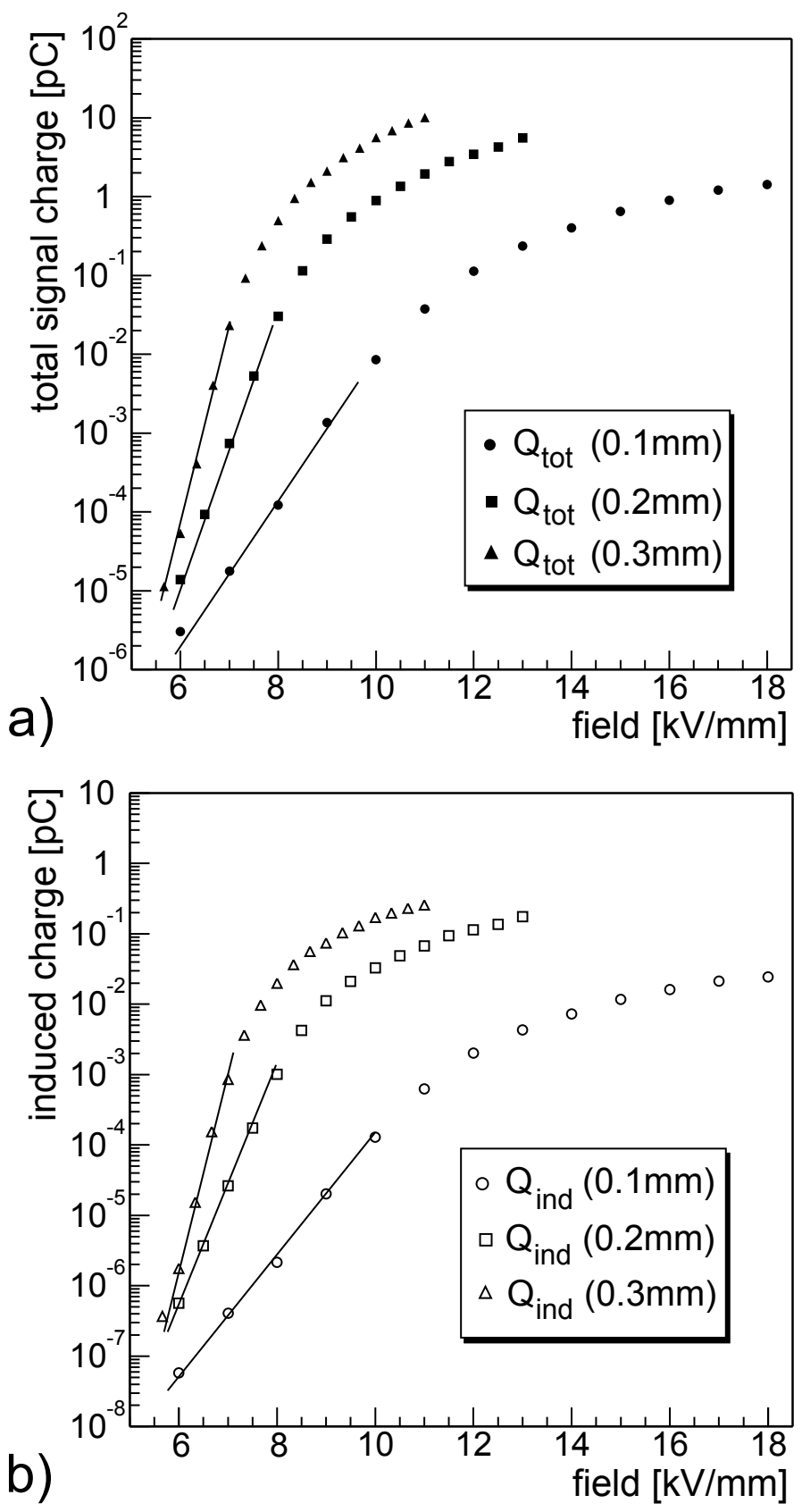

Figure 11: The average total signal charge (a) and average induced charge (b) of simulated avalanches in Timing RPCs with $0.1 \mathrm{~mm}$ to $0.3 \mathrm{~mm}$ gap widths versus the applied electric field. The solid lines are eye guides that correspond to exponential increase of the avalanche charges and evidence the sub-exponential character of the data at higher fields (gain saturation). 

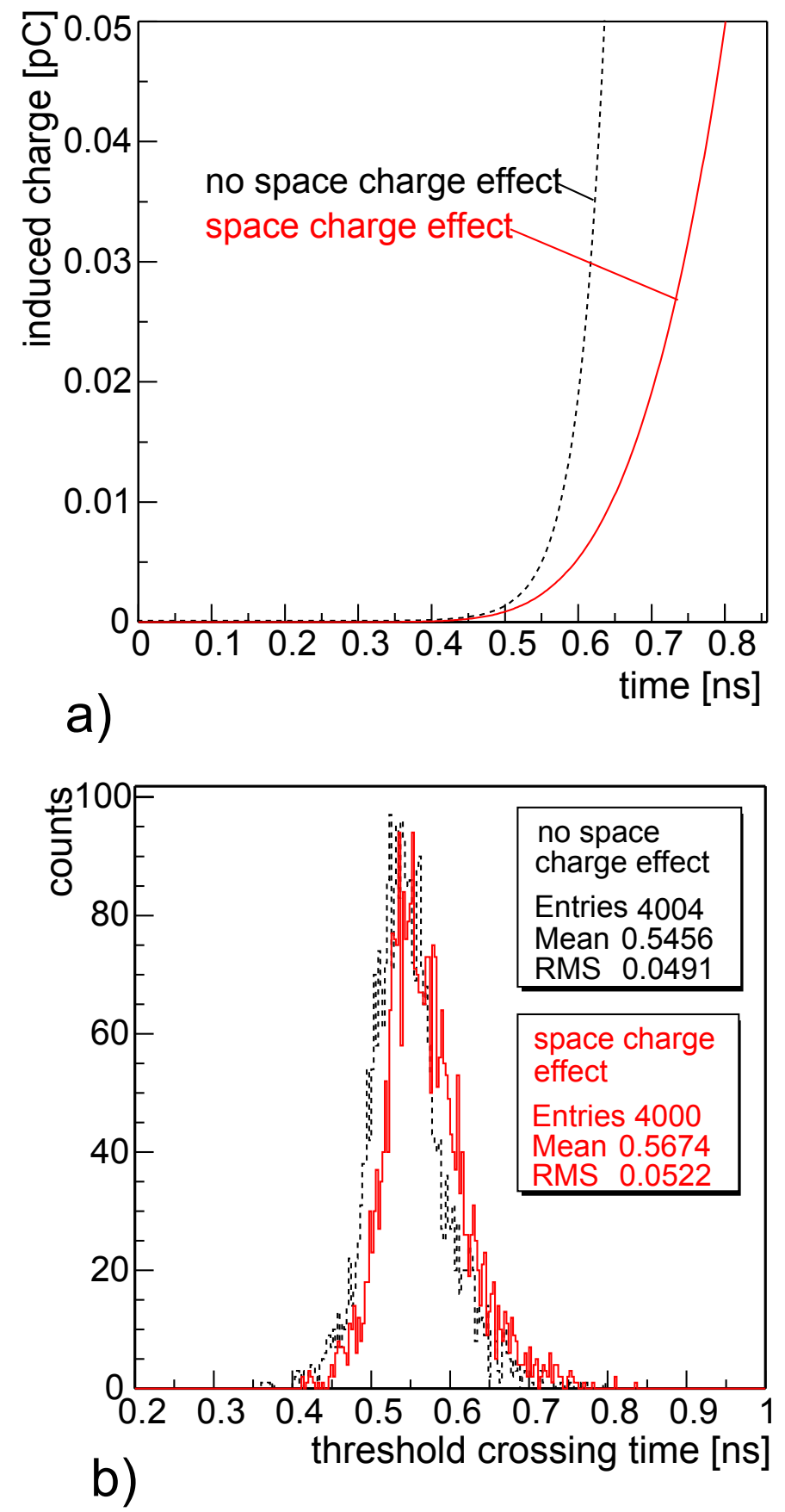

Figure 12: The influence of the space charge effect on the time resolution of Timing RPCs. We simulated a single gap Timing RPC at $3 \mathrm{kV}$, temperature $T=296.15 \mathrm{~K}$ and pressure $p=$ 970 mbar. a) A Comparison of simulated average signals at an early stage. We find that at the threshold level (typically $20 \mathrm{fC}$ ) the space charge effect does already influence the signal rise. b) Simulated intrinsic time resolutions (no amplifier). The time resolution is almost unaffected while the mean of the distribution is shifted slightly by the space charge effect. 


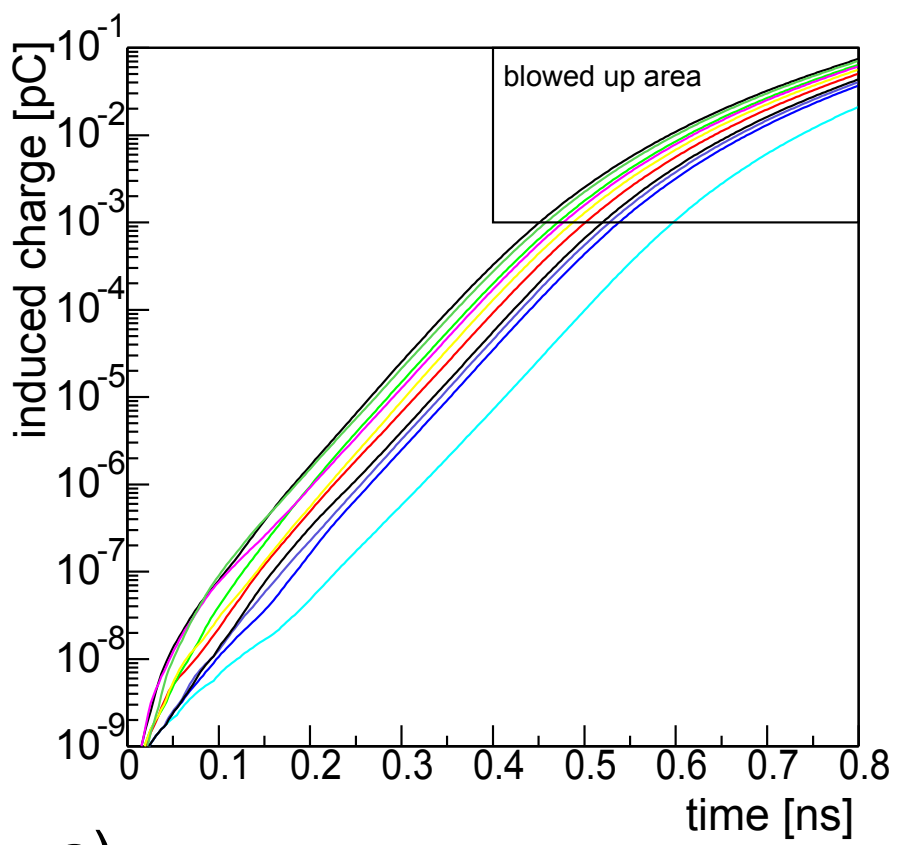

a)

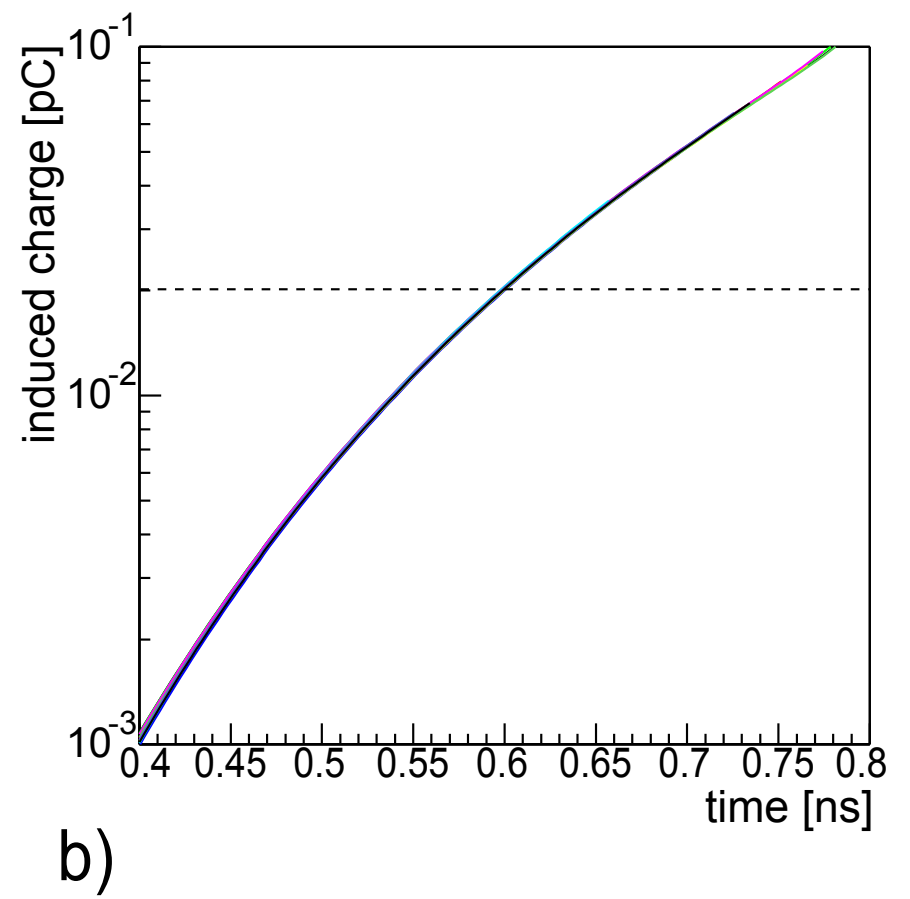

Figure 13: a) Some simulated avalanches in Timing RPCs. All avalanches were started by one electron in the same position. The fluctuation of the induced charge is large in the beginning when there are just a few electrons. At all later stages the avalanches grow similarly. b) A blow up of the indicated region above the threshold level of $10 \mathrm{fC}$. All 10 curves are shifted along the time axis so that they match at a threshold of $20 \mathrm{fC}$. We find that the shapes of the curves match very nicely. 


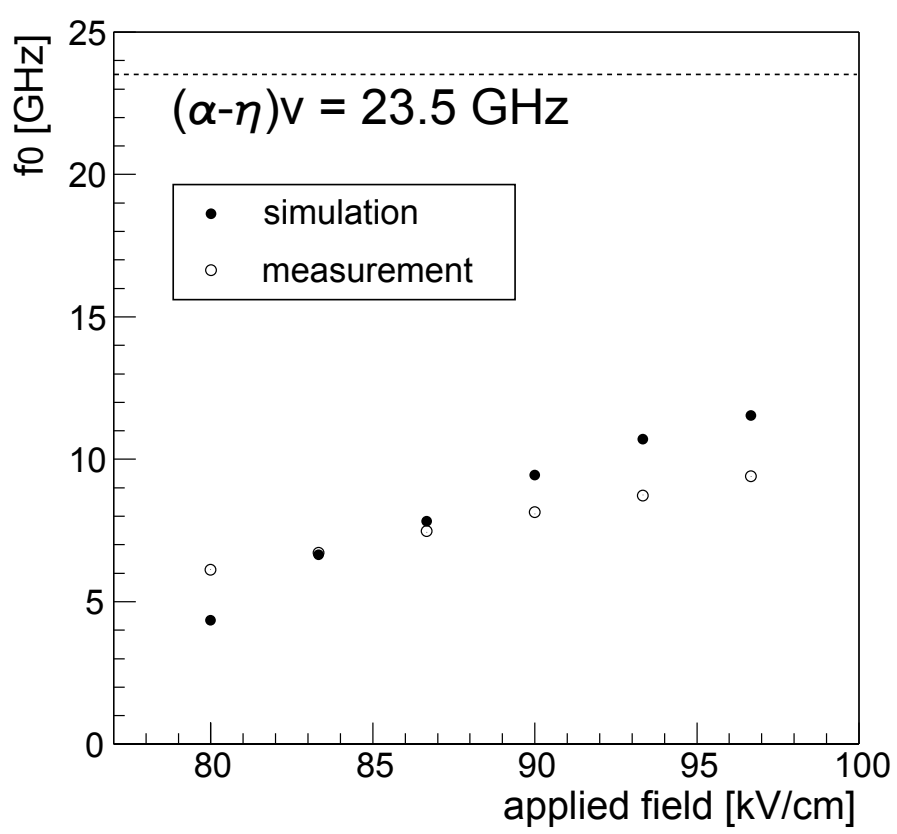

Figure 14: The value of $f_{0}=(\alpha-\eta) v_{D}$. We show simulated values (the solid circles) for $Q_{1}=$ $40 \mathrm{fC}$ and $Q_{2}=80 \mathrm{fC}$ and measurements (open circles) from [32].

it. We find that the mean is shifted slightly but the r.m.s. is almost unaffected. It seems that even though the space charge effect affects the signal rise time, its influence on the time resolution is negligible. To illustrate this result, we investigate single avalanches in Fig. 13, where the induced charge versus time is plotted for ten avalanches that were started by single electrons. The very beginning of the avalanche determines its final size [14]. This means that the fluctuation in the threshold crossing time, which determines the time resolution of an RPC, is caused by the avalanche fluctuations at a level where the avalanche is still small. An avalanche that is initially growing more rapidly reaches the threshold sooner than an avalanche that undergoes a slow initial growth. Once the avalanche has reached a sufficient size ( $\gtrsim 10 \mathrm{fC})$, it grows exponentially like $e^{(\alpha-\eta) z}$, if we neglect a space charge effect. Thus the avalanche growth is similar for different avalanches, once they have reached that size. The space charge effect introduces a deviation in the signal rise from an exponential (saturation), which explains the shift of the mean times. However, this deviation is similar for all signals so that the time resolution is not affected. If we overlay the different curves from Fig. 13a at the threshold level, which is done in Fig. 13b, we find that they are almost indistinguishable. This shows that the time resolution is determined by avalanche fluctuations at the very beginning and that space charge has no influence on it.

Finally we want to compare the signal rise times to measurements. An induced current signal would rise like $\exp \left(f_{0} t\right)$, where $f_{0}=(\alpha-\eta) v_{D}$, if no space charge effect is present. In [31] the authors show that sending this signal through a general linear network, the output signal shows the same exponential rise and $f_{0}$ can be measured by setting two thresholds $Q_{1}$ and $Q_{2}$ to the signal. From the two threshold crossing times $t_{1}$ and $t_{2}$ one finds $f_{0}$ by

$$
\ln \left(Q_{2} / Q_{1}\right)=f_{0}\left(t_{2}-t_{1}\right)
$$

This relation holds only if the input signal is exponential at the threshold crossing times. Fig. 14 shows measured [32] and simulated values of $f_{0}$ for different voltages in single gap Timing RPCs. The simulation is quite close to the measurement. The deviation of the measured 
and the simulated value of the expected value $f_{0}=(\alpha-\eta) v_{D}=23.5 \mathrm{GHz}$ is the consequence of the non-exponential growth of the avalanches at the threshold level due to the space charge effect, as it was plotted in Fig. 12.

\subsubsection{Charge-to-Time Correlation}

We now investigate detector-intrinsic charge-to-time correlations without electronics. In Fig. 15 we plot the time at which the threshold of $10 \mathrm{fC}$ is crossed by the signals versus the induced charge. We observe a triangular distribution that we will explain in the following. We will use the expression leading cluster, which refers to the cluster that is deposited at the position closest to the cathode. In general, the total signal charge is determined by the leading cluster because it has the longest drift distance and can thus reach the largest number of charge carriers. In Fig. 16 we show how the total signal charge is correlated to the position of the formation of the leading cluster. Obviously the signal charge is largest for avalanches with a leading cluster closer to the cathode. But let's come back to the charge-time correlation in Fig. 15. In the plot we have marked three zones that form the limits of the distribution:

Zone 1: The value of the threshold defines this limit. Avalanches that do not cross the threshold do not appear in the distribution.

Zone 2: Here we find the events with a very fast signal rise time. The fastest possible signal rise defines this limit. The signal rise time is determined by the avalanche statistics. As mentioned before, the very beginning of each avalanche determines its final size. Thus also the threshold crossing time is determined by the early stage of growth. In zone 2 we find no correlation of the threshold crossing time to the charge. To understand this we assume that the leading cluster determines the timing and that it is crossing the threshold very early. The position in the gas gap where it is deposited is randomly distributed. According to Fig. 16, the avalanche charge will be very high if it is deposited close to the cathode. Correspondingly the charge will be very low if it is deposited closer to the anode. In that case it may just be large enough to cross the threshold. As a consequence, the charge is not correlated to the time in this zone.

Zone 3: Here we find the events with a slow signal rise time. We observe a clear correlation of the threshold crossing time to the charge. Towards higher values of the total signal charge the signal rise is becoming faster. To understand this we again assume that the leading cluster determines the timing. The signals with the highest charges have a leading cluster that was deposited close to the cathode. They also have a fast signal rise time, meaning that the corresponding avalanches underwent a fast early growth. On the other hand the events with the slowest signal rise time are connected to avalanches with lower values of the total signal charge. This can be understood if one imagines that an avalanche with the leading cluster close to the cathode has to undergo a very slow growth process in order to arrive at a small final charge.

To further illustrate the charge-to-time correlation we simulated avalanches that were started by one electron either at random $z$-position in the gas gap or right at the cathode at $z=0$. Fig. 17 shows that for a given avalanche charge the signals with the slowest signal rise time are given by avalanches that are started at the (close to the) cathode. The highest charges are reached by avalanches starting at the (close to the) cathode and undergoing a fast initial growth process. 


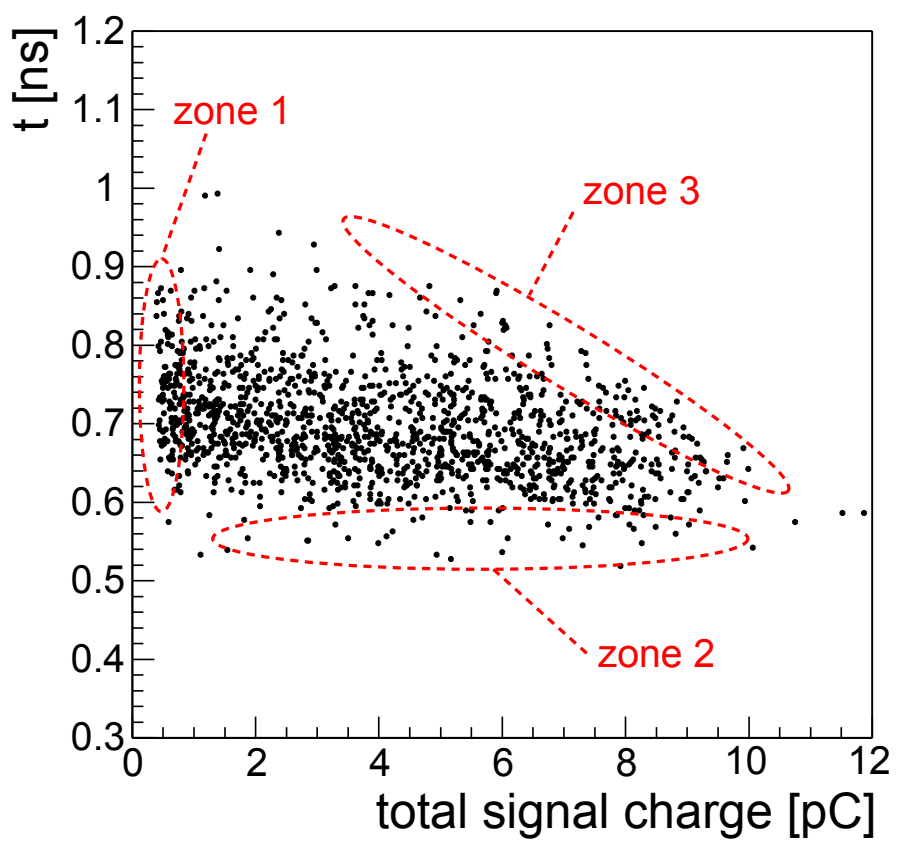

Figure 15: Intrinsic correlation of the charge threshold crossing time to the induced charge for simulated avalanches in a $0.3 \mathrm{~mm}$ single gap Timing RPC at $2.8 \mathrm{kV}$. The threshold applied to the induced charge was $10 \mathrm{fC}$. The temperature is $T=296.15 \mathrm{~K}$ and the pressure $p=970 \mathrm{mbar}$.

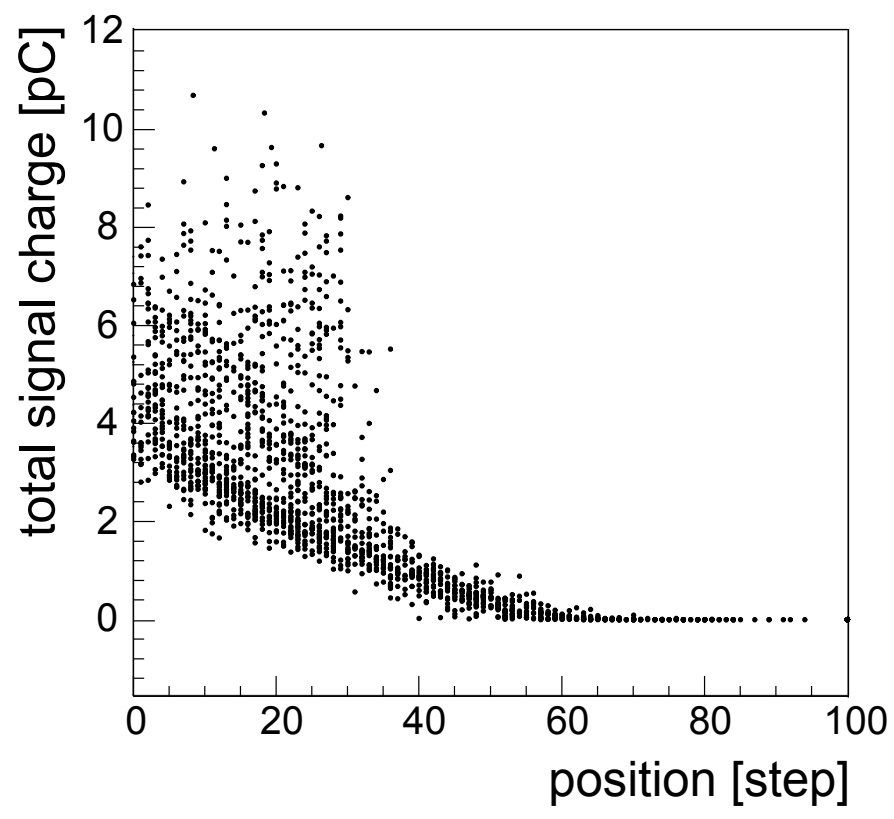

Figure 16: Correlation of the total signal charge to the position of the formation of the first cluster that reached the anode. We simulated avalanches in a $0.3 \mathrm{~mm}$ single gap Timing RPC at $2.8 \mathrm{kV}$. 


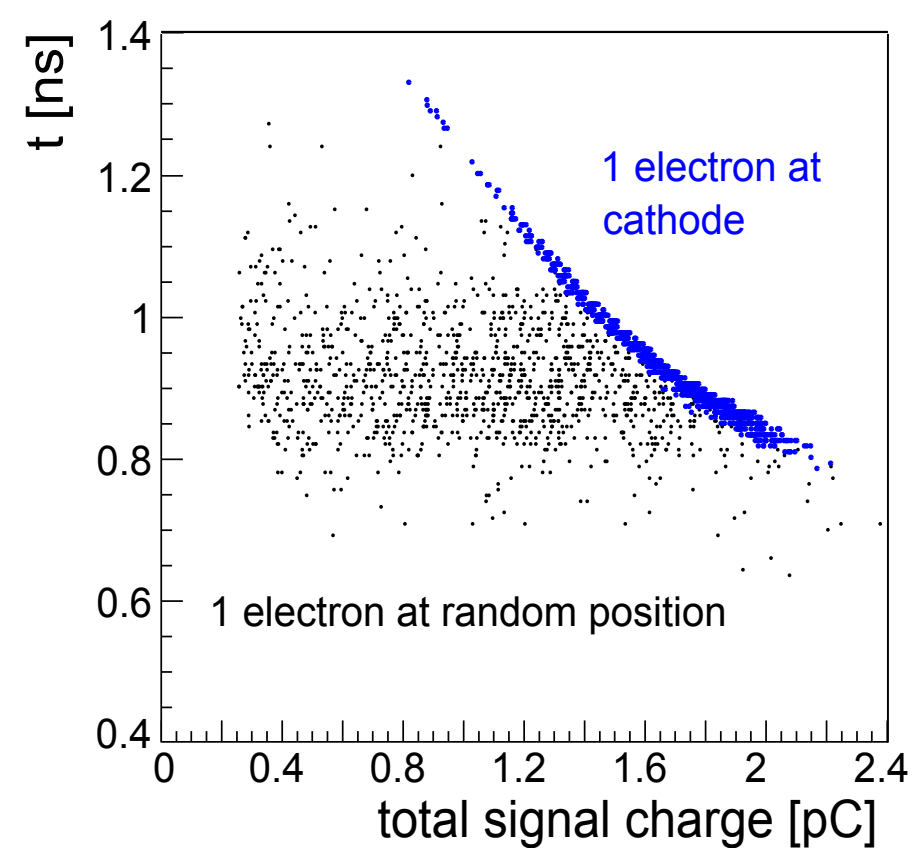

Figure 17: Simulated charge-time correlations for avalanches started by one electron, either at a random $z$-position in the gas gap or at the cathode at $z=0$.

\section{The 2-D Model}

Again we use cylindrical coordinates $r, z$ and $\phi$ and assume rotational symmetry of the avalanche around the z-axis. The simulation routine has the following basic structure:

1. A cylindrical volume of the gas gap is divided into a two dimensional grid of the $r$ and $z$-coordinates. If the $z$-coordinate is divided into $N_{z}$ steps of size $\delta z=g / N_{z}$, the corresponding time steps of the simulation are $\delta t=\delta z / v_{D}\left(E_{0}, p\right)$, where $v_{D}\left(E_{0}, p\right)$ is the electron drift velocity at the applied electric field $E_{0}$. The $r$-coordinate is divided into $N_{r}$ steps of an appropriately chosen size $\delta r$. The charge that is situated in the grid point $\left(r^{\prime}, z^{\prime}\right)$ is actually a charge ring of size $\delta r$ and $\delta z$ centered at the $z$-axis.

2. One electron is put inside the volume.

3. A two dimensional electric field vector $\left(E_{z}, E_{r}\right)$ at each bin is calculated, if there is at least one electron in that bin. The procedure is described in more detail in section 3.1.

4. The Townsend and attachment coefficients, the drift velocity and the diffusion coefficients at each bin are calculated according to Figs. 3 and 4.

5. The avalanches for each single electron are simulated according to the procedure described in [14]. The electrons are propagated. The procedure is described in section 3.2.

6. Steps 3 - 5 are repeated until all electrons have left the gas gap or got attached.

We assume a detector geometry as in Fig. 1b, where only one electrode is made of a resistive material and the other one is made of aluminum. We assume that the conductive electrode is the anode and that the cathode is made of $3 \mathrm{~mm}$ thick glass. Due to the conductivity of the anode, the electrons entering the anode plate disappear instantly. They do not contribute to the electric field in the gas gap.

\subsection{Calculation of the Electric Field Vector}

To calculate the electric field $\vec{E}_{\text {ring }}$ of a free charged planar ring containing the unit charge, we integrate over the electric field solution $\vec{E}_{\text {point }}$ of a free point charge in cylindrical coordinates: 


$$
\vec{E}_{\text {ring }}=\int_{0}^{2 \pi} \vec{E}_{\text {point }} d \phi^{\prime}
$$

$r^{\prime}, z^{\prime}$ give the radius and position of the ring and $(r, \phi, z)$ is the point of observation. Due to the rotational symmetry, we can choose $\phi=0$. We obtain

$$
\begin{aligned}
& E_{r}\left(r, z, r^{\prime}, z^{\prime}\right) \approx \frac{Q}{2 \pi \varepsilon_{2}} \frac{1}{r a^{2} b}\left[c^{2} E\left(\frac{-4 r r^{\prime}}{b^{2}}\right)+a^{2} K\left(\frac{-4 r r^{\prime}}{b^{2}}\right)\right], \\
& E_{\phi}\left(r, z, r^{\prime}, z^{\prime}\right)=0, \\
& E_{z}\left(r, z, r^{\prime}, z^{\prime}\right) \approx \frac{Q}{\pi \varepsilon_{2}} \frac{\left(z-z^{\prime}\right)}{a^{2} b} E\left(\frac{-4 r r^{\prime}}{b^{2}}\right),
\end{aligned}
$$

where

$$
\begin{aligned}
& a^{2}=\left(r+r^{\prime}\right)^{2}+\left(z-z^{\prime}\right)^{2}, \\
& b^{2}=\left(r-r^{\prime}\right)^{2}+\left(z-z^{\prime}\right)^{2}, \\
& c^{2}=r^{2}-\left(r^{\prime}\right)^{2}-\left(z-z^{\prime}\right)^{2}
\end{aligned}
$$

and

$$
K(x)=\int_{0}^{\frac{\pi}{2}} \frac{1}{\sqrt{1-x \sin ^{2}(\xi)}} d \xi \quad, \quad E(x)=\int_{0}^{\frac{\pi}{2}} \sqrt{1-x \sin ^{2}(\xi)} d \xi .
$$

$K(x)$ and $E(x)$ are the elliptic integrals of the first kind and of the second kind.

In the simulations we approximate the field of the space charge in the gas gap at the grid point $\left(z^{\prime}, r^{\prime}\right)$ with the sum of the fields of free charge rings (with radius $r^{\prime}$ at $z^{\prime}$ ) and of mirror charge rings on the anode side (with radius $r^{\prime}$ at $2 g-z^{\prime}$ ). The field of a mirror charge ring can be calculated by simply substituting $z^{\prime}$ with $2 g-z^{\prime}$ in Eqs. 7a and 7c. The field at $\left(r=r^{\prime}\right.$, $z=z^{\prime}$ ) is not included in the calculation of the space charge field due to the divergence of Eqs. $7 \mathrm{a}$ and $7 \mathrm{c}$ at this point.

\subsection{Propagation of the Charges}

Knowing the electric field strength at each grid point, the charges can be propagated. With the absolute value of the electric field $E=|\vec{E}(r, z)|=\sqrt{E_{r}^{2}(r, z)+E_{z}^{2}(r, z)}$ at the grid point $(r, z)$, we obtain the values of the Townsend coefficient $\alpha(E / p)$ and attachment coefficient $\eta(E / p)$, the drift velocity $v_{D}(E / p)$ and the longitudinal and transverse diffusion coefficients $D_{L}(E / p)$ and $D_{T}(E / p)$. As the next step, the electrons at each grid point are multiplied, according to the procedure described in [14]. The electrons are moved to a new grid point that lies in the direction of the drift velocity vector $\vec{v}_{D}$, which is parallel to the vector of the electric field $\vec{E}$. We also include diffusion here, keeping in mind that longitudinal diffusion is always in the direction of $\vec{E}$, which is not necessarily parallel to the $z$-axis. Accordingly, transverse diffusion is perpendicular to $\vec{E}$. In a coordinate system with $\tilde{x}, \tilde{y}, \tilde{z}$ and with the $\tilde{z}$-axis parallel to $\vec{E}$, the propagation and diffusion are calculated the following: 
- The new $\tilde{x}$-coordinate is calculated by drawing a random number from a Gaussian distribution with mean $\mu=0$ and sigma $\sigma=D_{T} \sqrt{\delta l}=D_{T} \sqrt{\left|v_{D}\right| \delta t}$. Here $\delta l$ is the drifted distance and $\delta t$ is the time step of the simulation.

- The new $\tilde{y}$-coordinate is calculated accordingly.

- The new $\tilde{z}$-coordinate is calculated by drawing a random number from a Gaussian distribution with mean $\delta l$ and sigma $\sigma=D_{L} \sqrt{\delta l}=D_{L} \sqrt{\left|v_{D}\right| \delta t}$.

Since the electric field has in the main coordinate system the direction $\theta$, the propagation has to be rotated. For $\theta$ we find the relations

$$
\cos (\theta)=\frac{E_{z}}{|E|} \quad \text { and } \quad \sin (\theta)=\frac{E_{r}}{|E|}
$$

Since the problem has cylindrical symmetry we can chose $x=\tilde{x}$. The rotation is then performed by

$$
\left(\begin{array}{l}
x \\
y \\
z
\end{array}\right)=\left(\begin{array}{ccc}
1 & 0 & 0 \\
0 & \cos (\theta) & \sin (\theta) \\
0 & -\sin (\theta) & \cos (\theta)
\end{array}\right)\left(\begin{array}{l}
\tilde{x} \\
\tilde{y} \\
\tilde{z}
\end{array}\right)
$$

With the new coordinates $r=\sqrt{x^{2}+y^{2}}$ and $z$ the electrons are redistributed onto the bins. For large numbers of electrons this procedure becomes very time consuming. In that case the electrons are propagated in groups.

\subsection{Comparison of the different models}

In this section we compare the results of the two different simulation models. Figs. 18a and $18 \mathrm{~b}$ show the development of the total number of electrons in random avalanches started by one electron at the cathode. We observe that the initial growth is very similar for the different models. The avalanches reach their maxima at the same time step. At the stage where the number of electrons is large, the growth rate is suppressed to a larger extend in the avalanche that is simulated with the 1.5D model. Even though the initial growth of this avalanche was stronger, the maximum number of electrons is only around a third of the number that is obtained with the 2-D model. The saturation effect is stronger in the 1.5-D case. Two reasons can be given:

- In the 1.5-D model we calculate the $z$-component of the electric field of radial Gaussian charge distributions, namely always at $r=0$, in the center of the avalanche. However, here the field has the largest value. In a real avalanche electrons are also situated at other positions with $r \neq 0$, where the field is less strong. This means that in the 1.5-D simulation we calculate a space charge field that is somewhat too strong.

- The electron clouds are distorted transversely by the attractive and/or repulsive space charge fields. In the 1.5-D model, on the other hand, the standard deviations $\sigma$ of the radial Gaussian charge distributions depend only on the transverse diffusion. If we accept that the radial repulsion of the electrons in the center and at the tip of the electron clouds leads to an increase of the radial spread of the avalanche, then $\sigma$ is chosen too small. Thus we will overestimate the radial charge density in the 1.5 -D model. The $z$-component of the electric field of radial Gaussian charge distributions with different $\sigma$ can differ considerably. As as a result, the space charge effect is overestimated in the 1.5-D model. After the maximum is reached, the electron number decreases due to attachment and due to the fact that electrons enter the anode and leave the gas gap (compare to Fig. 9). The decline 

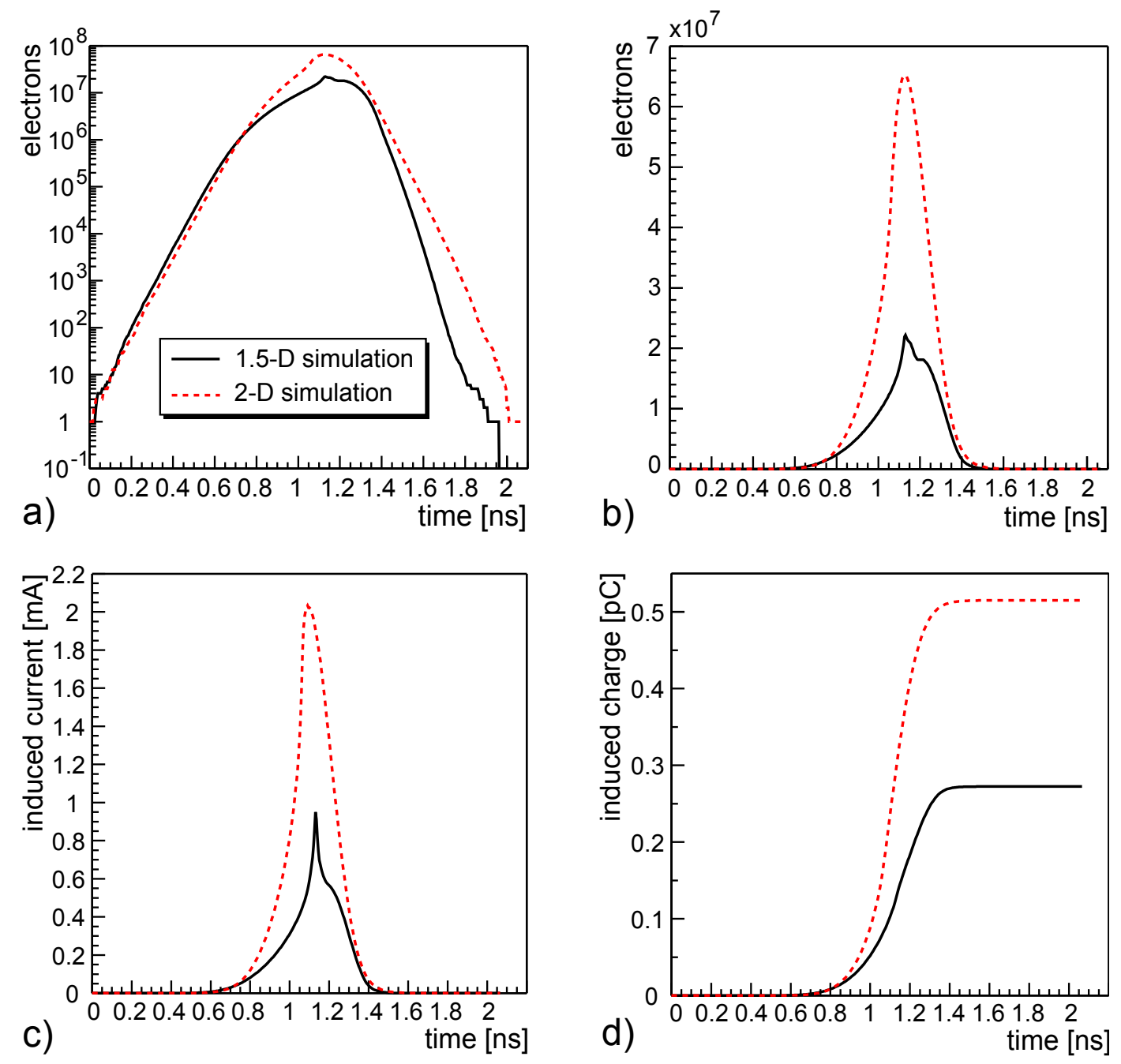

Figure 18: Comparison of two random avalanches that were simulated with the different models that implement the space charge effect. We simulated a Timing RPC at a high voltage of $2.8 \mathrm{kV}$ filled with the standard gas mixture $\mathrm{C}_{2} \mathrm{~F}_{4} \mathrm{H}_{2} / \mathrm{i}-\mathrm{C}_{4} \mathrm{H}_{10} / \mathrm{SF}_{6}(85 \%, 5 \%, 10 \%)$ at $T=296.15 \mathrm{~K}$ and $p=1013$ mbar. The avalanches were started by one electron at the cathode. In both models we used a division of the $z$-axis into 300 steps. In the 2D model the $r$-coordinate is divided in 150 steps. a) The total number of electrons versus time. b) The same plot on a linear scale. c) The induced current versus time. d) The induced charge versus time. 
of the electron number is faster in the case of the 1.5-D model. Here propagation is only allowed longitudinally along the $z$-axis. Since the calculated space charge fields are stronger in the 1.5-D model than in the 2-D model, the region with strong attachment is larger in the 1.5-D case.

The induced current signal and the development of the induced charge are shown in Figs. $18 \mathrm{c}$ and $18 \mathrm{~d}$. The charges that are induced by the avalanches are $0.27 \mathrm{pC}$ for the $1.5-\mathrm{D}$ model and $0.52 \mathrm{pC}$ for the 2-D model. The difference is about a factor of two. The charge of the ions in the gas gap at the time when the electrons have all either entered the anode or got attached ${ }^{2}$ is $1.79 \mathrm{pC}$ for the $1.5-\mathrm{D}$ model and $4.05 \mathrm{pC}$ for the $2-\mathrm{D}$ model.

\subsection{Results for Timing RPCs}

\subsubsection{Electron Density}

Fig. 19 shows the electron distribution in an avalanche that was started by a single electron at position $r=0, z=0$ and at $t=0 \mathrm{~ns}$. From the cathode the electron crosses the whole gap and reaches a maximum size of almost $4 \times 10^{7}$ electrons.

(a) On the first image at $t=1.0 \mathrm{~ns}$ we observe that the shape of the distribution differs from a strictly Gaussian shape. It is very similar to the shapes that were obtained with the 1.5-D model (see Fig. 9). At the tip of the distribution the multiplication is very strong while at the tail it is less strong.

(b) At $t=1.05 \mathrm{~ns}$ the peak of the electron distribution reaches the anode. The actual drift velocity in the gas and at the applied field strength would lead to a drift time of $t_{D}=$ $0.3 \mathrm{~mm} /(0.2 \mathrm{~mm} / \mathrm{ns})=1.5 \mathrm{~ns}$. Nevertheless, diffusion combined with the repulsive space charge field that acts on the electrons at the tip of the distribution lead to an acceleration of the electrons there.

(c) At $1.19 \mathrm{~ns}$ many electrons have already entered the conductive anode. The shape of the distribution changes dramatically from now on.

(d) At $1.29 \mathrm{~ns}$ the electrons close to the anode disappear rapidly. They are attached to the electronegative gas components due to the lowered field in this region.

$(e, f)$ The electrons leave the gas gap or get attached. The electron density in the center of the avalanche at small values of $r$ decreases faster than further 'outside'.

Fig. 20 shows a contour plot of the electron density in a cluster of electrons that moves towards the anode at $z=0.03$. While in the first image at $t=0.5 \mathrm{~ns}$, where the avalanche is still relatively small ( $\approx 8 \times 10^{5}$ electrons), the shape of the distribution is roughly symmetric, it is different in the second image $(t=0.65 \mathrm{~ns})$. Here the avalanche has reached a size of about $1.3 \times 10^{7}$ electrons and the electrons at the tip of the electron cloud are repelled transversely from the center at $r=0$ by repulsive space charge fields, while at the tail of the distribution they are attracted by a field of opposite sign. We shall investigate the radial space charge field in more detail in a later section.

\subsubsection{Total Ion Density}

We now focus on the ion distribution. Fig. 21 shows the total ion density in an avalanche that was started by a single electron at position $r=0, z=0$ and at $t=0 \mathrm{~ns}$. We show the number of positive ions minus the number of negative ions at each grid point.

(a) The shape of the distribution of ions at $t=0.71 \mathrm{~ns}$ represents an approximately exponential avalanche growth combined with diffusion. The avalanche consists of about $1 \times 10^{6}$ electrons.

2) This is the number of positive ions minus the number of negative ions. It is not to be confused with the total signal charge $Q_{t o t}$ that is given by the number of positive ions. 

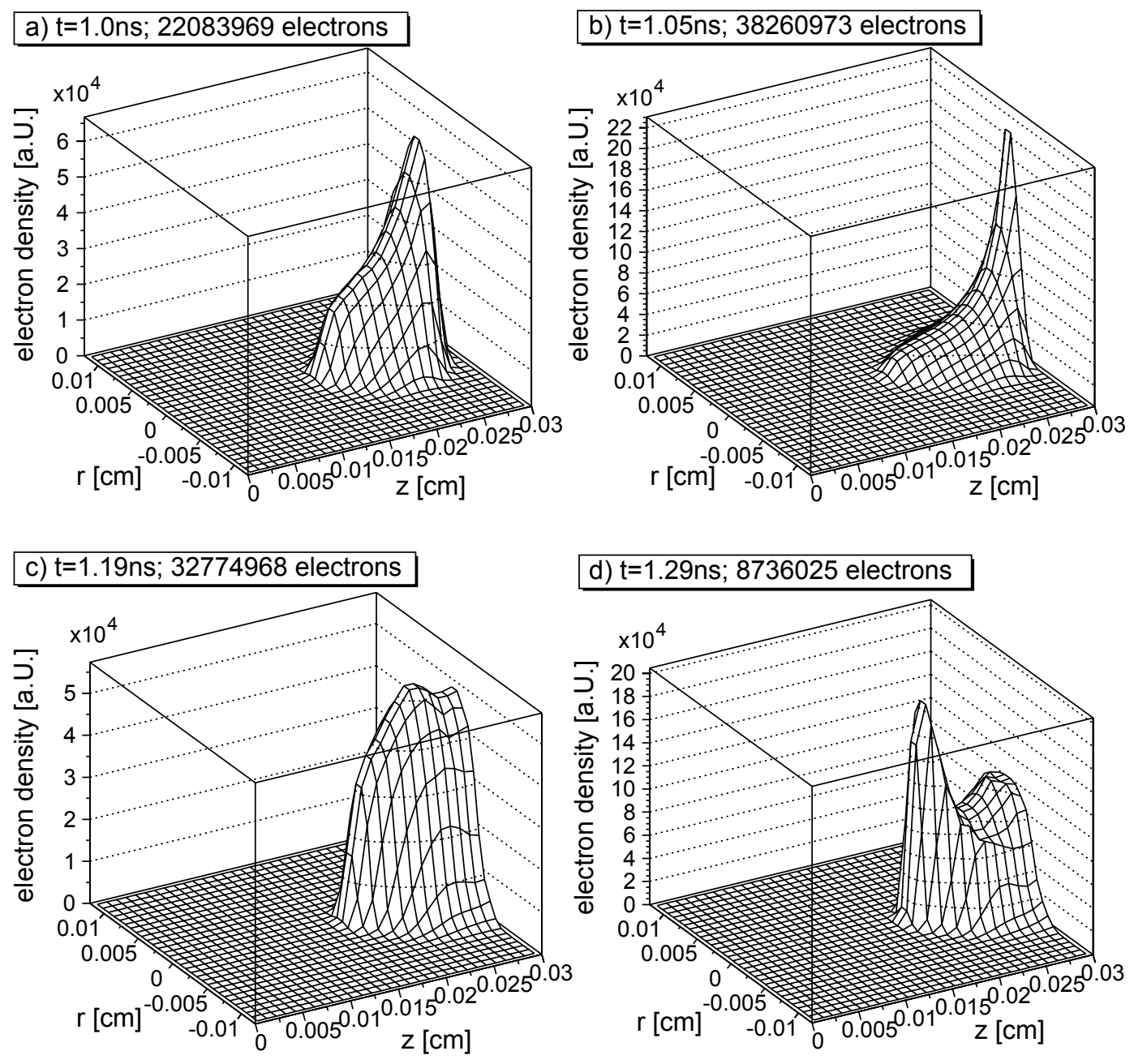

e) $t=1.38 n s ; 1134153$ electrons

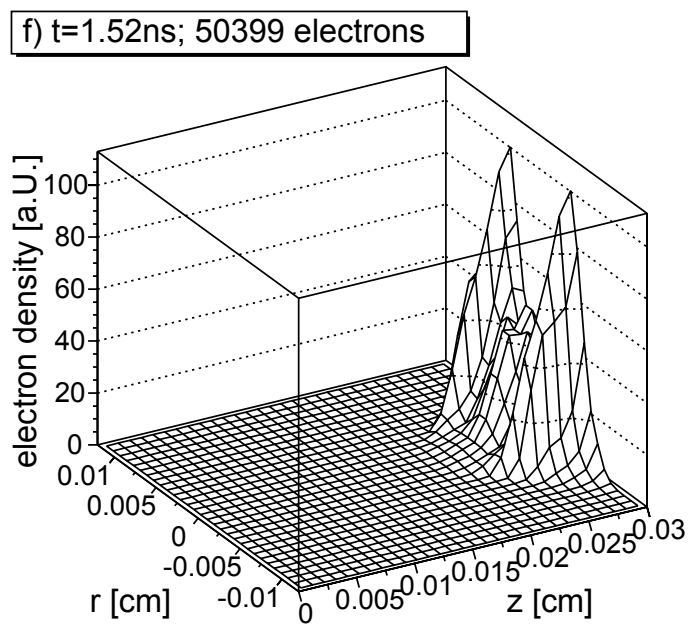

Figure 19: Electron density in an avalanche in a $0.3 \mathrm{~mm}$ gap Timing RPC filled with the standard gas mixture and with an operating voltage of $2.8 \mathrm{kV}$. The avalanche was started by one electron at $(r=0 \mathrm{~mm}, z=0 \mathrm{~mm})$. 

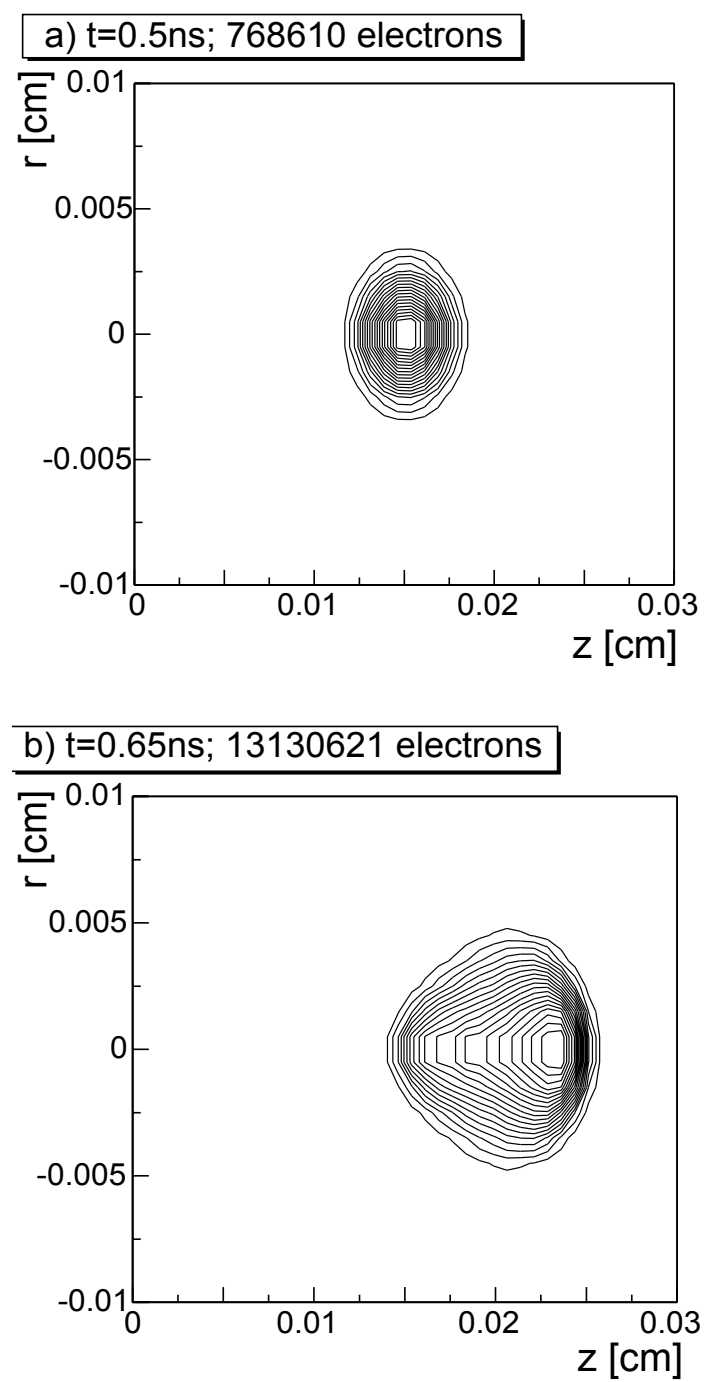

Figure 20: Electron density in an avalanche in a $0.3 \mathrm{~mm}$ gap Timing RPC filled with the standard gas mixture and with an operating voltage of $2.8 \mathrm{kV}$.

(b) At $t=0.81 \mathrm{~ns}$ the shape is different. As the electron cloud propagates, the multiplication and thus the increase of the number of ions is smaller than before. The same effect was found in the 1.5-D simulations (see Fig. 9).

(c) At $1.05 \mathrm{~ns}$ the avalanche has reached the anode. The shape of the ion distribution is very similar to the shape of the electron distribution as shown in image $19 \mathrm{~b}$.

(d) At $1.14 \mathrm{~ns}$ the number of positive ions close to the anode decreases rapidly, which is due to the attachment of electrons in that region (compare with Fig. 19).

(e) At $1.24 \mathrm{~ns}$ the number of negative ions grows further, leading to a reduction of the density of positive ions.

(f) At $1.95 \mathrm{~ns}$ most electrons either entered the anode or got attached. The density of positive and negative ions close to the anode is similar so that the overall ion density approaches zero here (compare to Fig. 9).

\subsubsection{Electric Field}

The value of the electric field $E(r, z, t)=|\vec{E}(r, z, t)|$ sensed by the electrons in the gas gap determines the values of the gas parameters (Townsend and attachment coefficients, 

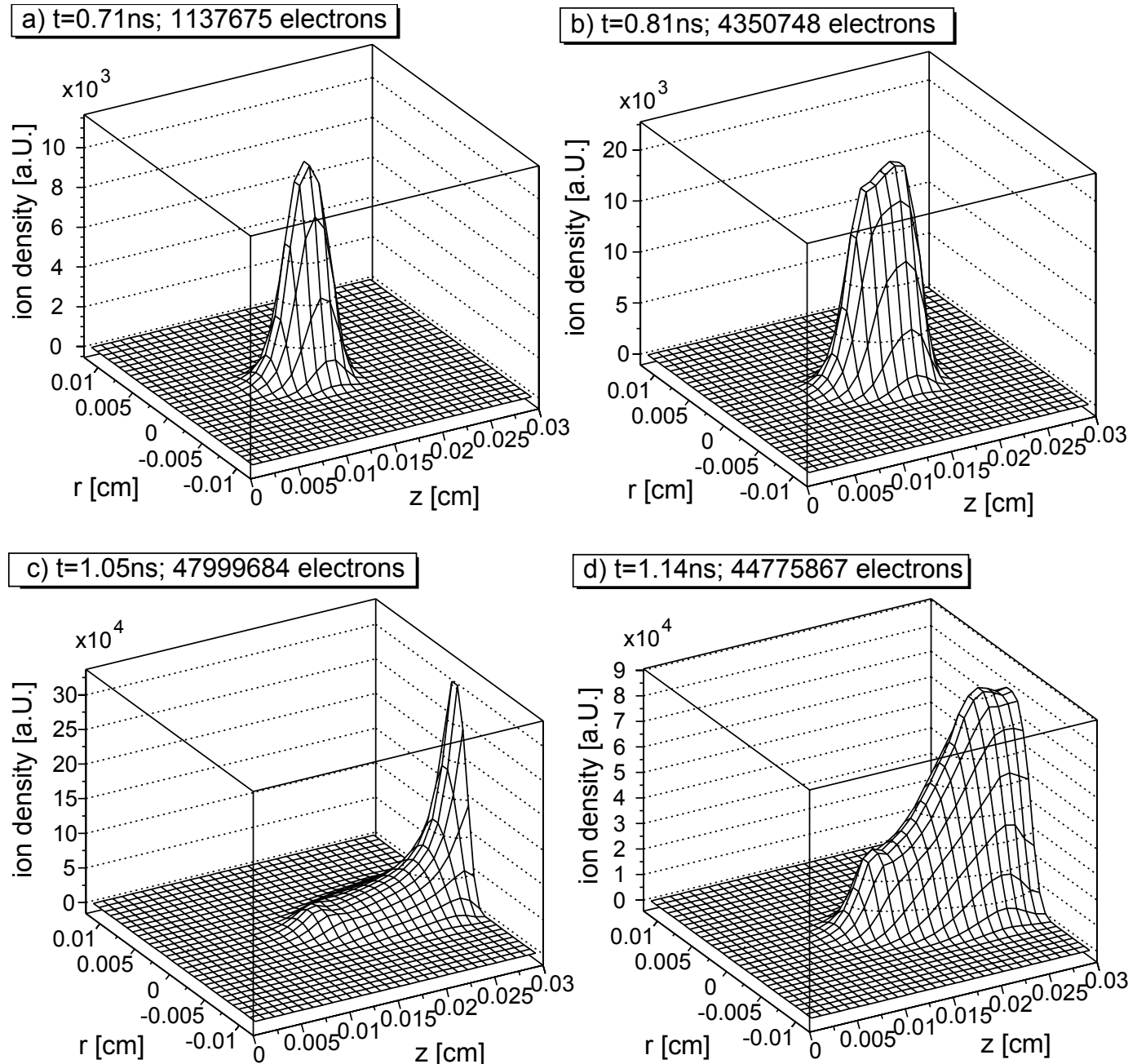

e) $t=1.24 n s ; 16629278$ electrons
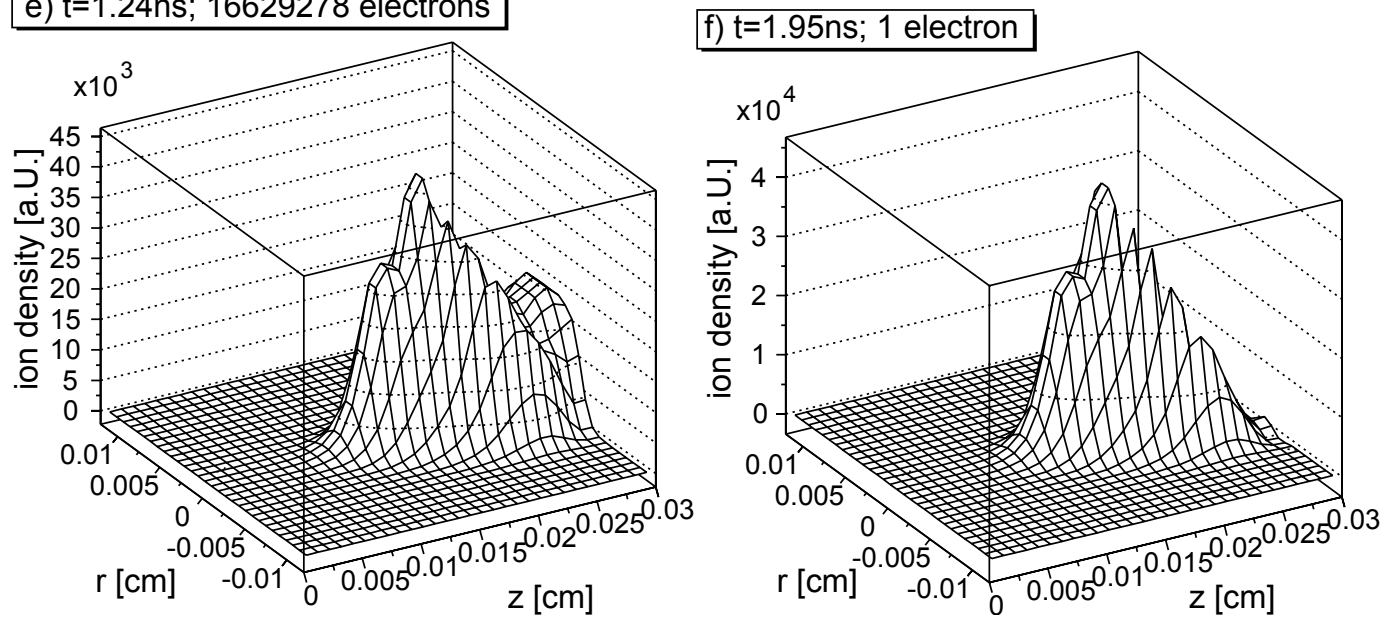

Figure 21: Ion density (number of positive ions minus number of negative ions) in an avalanche started by one electron at ( $r=0, z=0)$ in a Timing RPC at an operating voltage of $2.8 \mathrm{kV}$. 

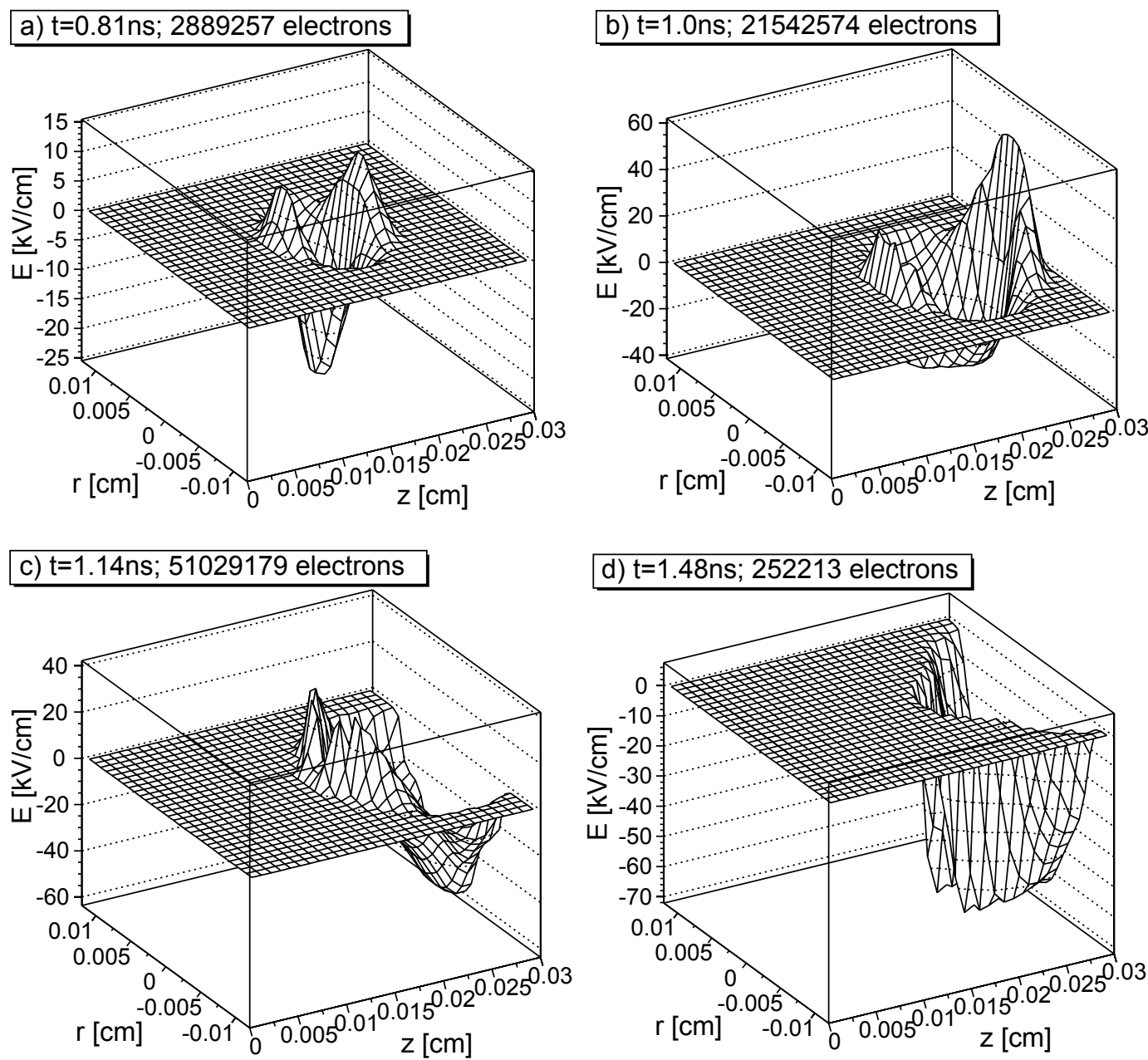

Figure 22: Absolute value of the space charge field. The field is set zero at all grid points where no electrons are situated.

diffusion coefficients and drift velocity). Fig. 22 shows the absolute value of $E(r, z, t)$ at different times and at the different grid points where electrons are situated. Again we simulated an avalanche that was started by a single electron at the position $r=0, z=0$ and at $t=0 \mathrm{~ns}$. We show the value of the electric field contributed by the avalanche charges (the space charge field). The total field can be calculated by adding the applied external electric field $E_{0}=93.3 \mathrm{kV} / \mathrm{cm}$.

$(a, b)$ In the initial phase of the avalanche, where the electron cloud has not yet reached the anode (at $t=0.81 \mathrm{~ns}$ and at $t=1.0 \mathrm{~ns}$ ), the electrons are distributed both in regions with increased and lowered electric field strength. While at the tip, where the field can be increased dramatically, the electron density is large (compare to Fig. 19a), there are only a few electrons at the tail, where the field is increased as well.

(c) At a later stage (at $t=1.14 \mathrm{ns),} \mathrm{where} \mathrm{the} \mathrm{avalanche} \mathrm{has} \mathrm{reached} \mathrm{the} \mathrm{anode} \mathrm{and} \mathrm{consists} \mathrm{of}$ about $5.1 \times 10^{7}$ electrons, the field is lowered almost everywhere, where we find electrons. But still we find a region at the tail of the electron cloud, where electrons sense an electric field strength that is increased by up to $40 \%$.

(d) The electrons then all enter the region of decreased electric field strength in front of the anode. At $t=1.48 \mathrm{~ns}$, where the avalanche has $5.1 \times 10^{5}$ electrons, and at all later stages, the electrons will sense an accordingly lowered drift velocity and effective Townsend coefficient. 
From this data we learn that there are regions of drastically decreased electric field strength in an avalanche that are the cause of the saturated growth and the low observed final avalanche charges. We also observe regions where the electric field is increased dramatically, but there is only a minority of electrons situated in those regions. The increased multiplication at the regions of higher field strength is more than compensated by the dramatically decreased multiplication in the center of the electron clouds.

\subsubsection{Effective Townsend Coefficient}

In this section we investigate the value of the parameter that determins the avalanche multiplication during the evolution of an avalanche in the gas gap of a Timing RPC: the effective Townsend coefficient $\alpha_{e f f}(r, z, t)=\alpha(E(r, z, t) / p)-\eta(E(r, z, t) / p)$. Fig. 23 shows the value of $\alpha_{e f f}(r, z, t)$ at different times and at the different grid points given by the $r$ - and $z$-coordinates. Again we simulated an avalanche that was started by a single electron at the position $r=0, z=0$ and at $t=0 \mathrm{~ns}$. A positive (negative) effective Townsend coefficient means that on average the number of electrons in the corresponding grid point will increase (decrease). We call $\alpha_{0}$ the value of $\alpha_{e f f}(r, z, t)$ at the applied electric field strength $E_{0}$.

(a) At $t=0.95 \mathrm{~ns}$ the avalanche has almost reached the anode and consists of around $1.9 \times$ $10^{7}$ electrons. The deformations in the distribution of the effective Townsend coefficient in the gas gap are very large. At the tip (tail) of the electron cloud the increase is more than $100 \%(50 \%)$ as compared to $\alpha_{0}$, in the center the effective Townsend coefficient is just negative.

(b) At $t=1.0 \mathrm{~ns}$ the first electrons have reached the anode. Now the effective Townsend coefficient reaches a value of three times $\alpha_{0}$. In the center of the electron distribution, where most of the $3.0 \times 10^{7}$ electrons are situated, the value of $\alpha_{e f f}(r, z, t)$ is negative.

(c) At $t=1.1 \mathrm{~ns}$ the avalanche has grown to about $6.1 \times 10^{7}$ electrons. Up to now at most positions the value of $\alpha_{\text {eff }}(r, z, t)$ was still positive. Now we find a region in front of the anode where $\alpha_{\text {eff }}(r=0, z \rightarrow g, t)<-1000 / \mathrm{cm}$.

(d) At $t=1.24 \mathrm{~ns}$ most electrons are situated in the region of very low effective Townsend coefficient in front of the anode. In the largest fraction of this region the value of $\alpha_{e f f}(r, z, t)$ is negative. The minimum value is around $-1500 / \mathrm{cm}$. Accordingly, the number of electrons has dropped to $2.3 \times 10^{7}$. However, in the region further away from the anode, we still find a region of strong multiplication with an effective Townsend coefficient up to $150 \%$ increased as compared to $\alpha_{0}$. As was mentioned earlier, in this region the electron density is small so that the effect on the avalanche growth at this stage is small.

(e) At $t=1.31 \mathrm{~ns}$ the number of electrons has dropped to $1.9 \times 10^{6}$ electrons. Almost everywhere the effective Townsend coefficient is much lower than $\alpha_{0}$. The minimum value reaches $-6000 / \mathrm{cm}$ !

(f) At $t=1.62 \mathrm{~ns}$ the few electrons left in the gas gap undergo strong attachment. The minimum value of $\alpha_{e f f}(r, z, t)$ is around $-2000 / \mathrm{cm}$.

\subsubsection{Radial Electric Field}

The value of the $r$-component of the electric field $E_{r}(r, z, t)$ that is sensed by the electrons in the gas gap is the parameter that determines the radial spread of the electron cloud on top of the transverse diffusion. Fig. 24 shows the value of $E_{r}(r, z, t)$ at different time steps and at the different grid points where electrons are situated. Again we simulated an avalanche that was started by a single electron at position $r=0, z=0$ and at $t=0 \mathrm{~ns}$.

(a) $t=0.81 \mathrm{~ns}$ : At the early stages of the avalanches, where no electrons have yet reached the anode, the electrons are attracted (repelled) to (from) the center of the avalanche at 

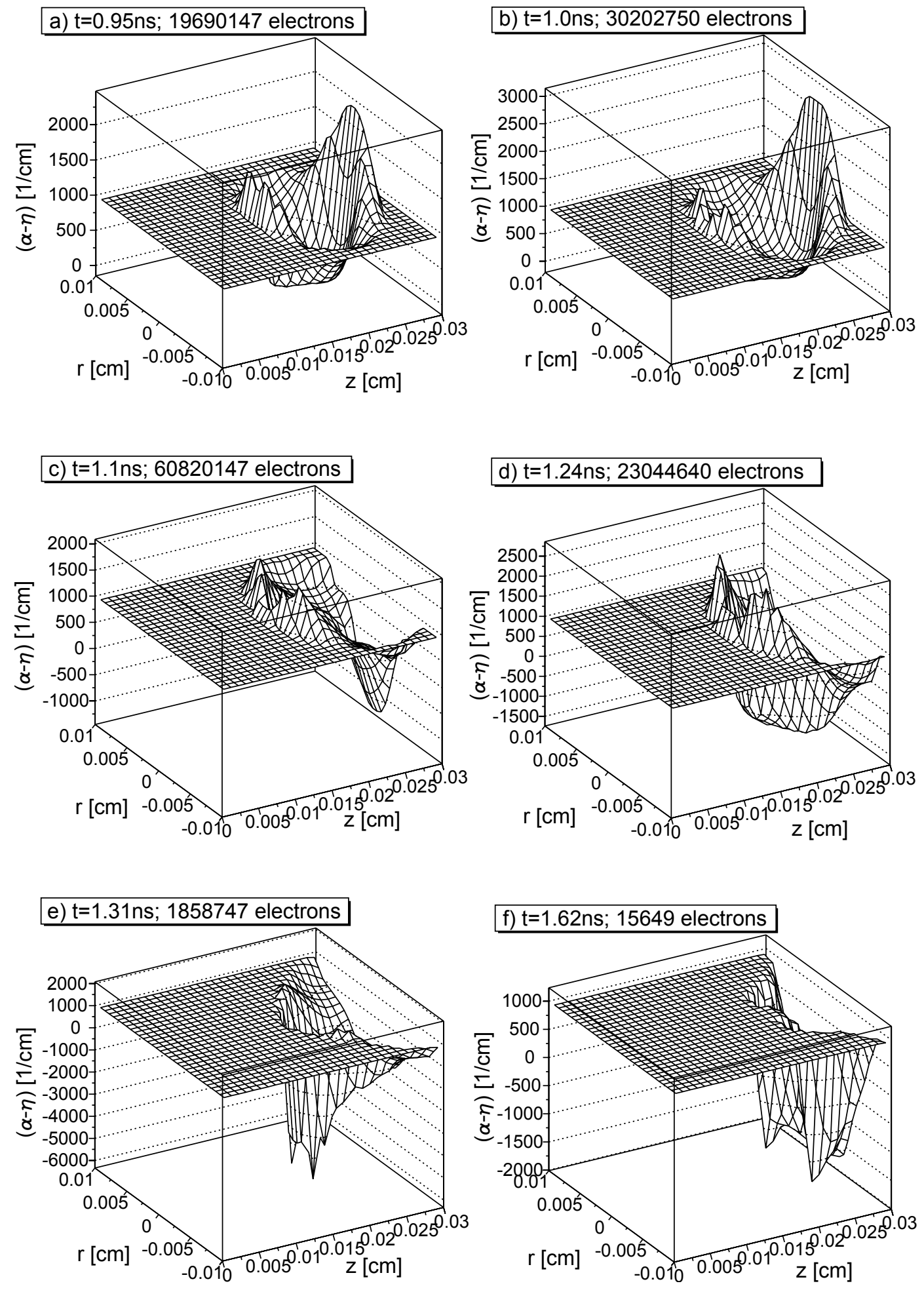

Figure 23: Effective Townsend coefficient at the positions where electrons are situated in the gas gap. 

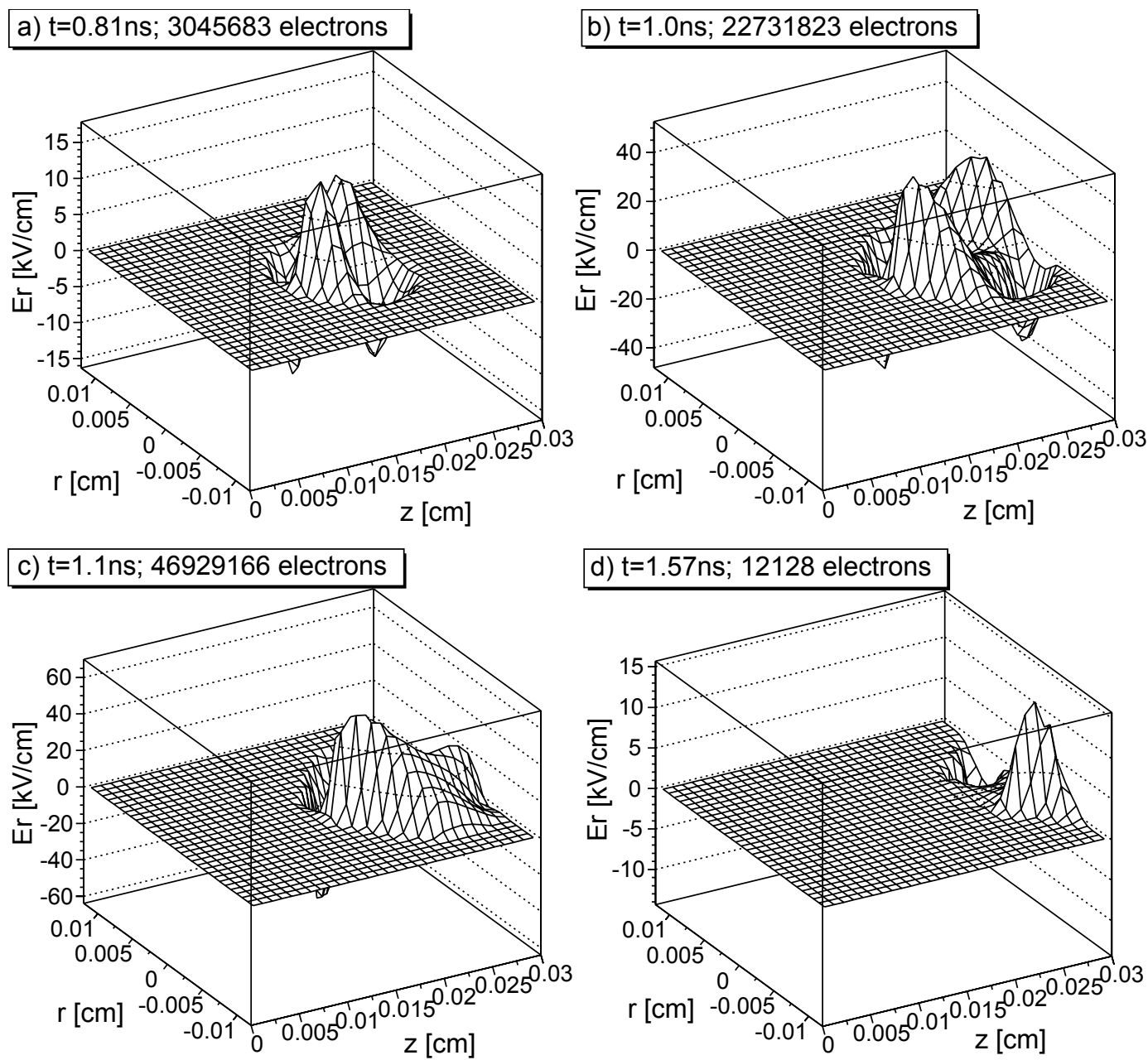

Figure 24: $r$-component of the space charge field. The field is set zero at the points where no electrons are situated.

$r=0$ at the tip (tail) of the avalanche (compare to Fig. 20).

(b) $t=1.0 \mathrm{~ns}$ : The first electrons reach the anode.

(c) $t=1.1 \mathrm{~ns}$ : As many free electrons have left the gas gap, the density of positive ions now is larger than the density of electrons. The field now attracts electrons to the center everywhere.

(d) $t=1.57 \mathrm{~ns}$ : The value of $E_{r}(r, z, t)$ is decreasing.

Generally we observe that the radial space charge field reaches values that are comparable to the longitudinal space charge field.

\section{$4 \quad$ Summary and Conclusions}

We have applied standard detector physics simulations to RPCs and find good agreement with measurements. While from the simple electric field configuration found in the gas gap of an RPC one might expect a just as simple description of the avalanche propagation, it turns out that the physical processes are very complex. The detailed simulation of the signal development demands for the dynamic calculation of the electric field that is sensed by the electrons in the avalanche and that is contributed by the positive and negative avalanche charges. Especially at the final stages of the avalanche development this space charge field can easily reach the same strength as the applied electric field. We can roughly divide this field into three zones: In the 
two zones at the tip and at the tail of the electron distributions the total electric field is increased by the space charge field. At the center of the avalanche, where most of the electrons are situated, the total electric field is strongly decreased. Assuming an avalanche in an electronegative gas, we find negative values for the effective Townsend coefficient and thus strong attachment here. At a later stage, when the electron cloud has reached the anode, almost all electrons are situated in that zone of extremely lowered field strength and attachment dominates. Moreover, we find that the radial electric field contributions of the space charge are within the same order of magnitude as the applied electric field. Thus the influence of this effect on the radial spread of the avalanches at large gas gain is large.

The detector behaviour is well understood. The experimentally observed average avalanche charges and the shapes of charge spectra can be explained with standard detector physics and the values of the gas parameters as predicted by HEED, MAGBOLTZ and IMONTE. Contrary to wire chambers, RPCs operate in a strong space charge mode. The experimental result of a first exponential and then linear dependence of the average charges on the high voltage is reproduced by the simulations. The space charge effect affects the signal rise already at the threshold level but has no influence on the time resolution.

\section{References}

[1] R.Santonico and R. Cardarelli, Development of resistive plate counters, Nucl. Instr. and Meth. 187 (1981) 377-380

[2] R.Santonico and R. Cardarelli, Progress in resistive plate counters, Nucl. Instr. and Meth. A 263 (1988) 20-25

[3] ATLAS Muon Spectrometer Technical Design Report, ATLAS TDR 10, CERN-LHCC97-22, 1997

[4] CMS Muon Technical Design Report, CMS TDR 3, CERN/LHCC 97-32. 1997

[5] P. Fonte, A. Smirnitski and M.C.S. Williams, A new high resolution TOF technology, Nucl. Instr. Meth. A 443 (2000) 201-204

[6] E. Cerron Zeballos et al., A new type of resistive plate chamber: the multigap RPC, Nucl. Instr. Meth. A 374 (1996) 132-136

[7] A. Akindinov et al., The multigap resistive plate chamber as a time-of-flight detector, Nucl. Instr. Meth. A 456 (2000) 16-22

[8] P. Fonte et al., High-resolution RPCs for large TOF systems, Nucl. Instr. and Meth. A 449 (2000) 295-301

[9] A. Akindinov, P. Fonte et al., A four-gap glass-RPC time-of-flight array with 90ns time resolution, CERN-EP 99-166, 1999

[10] P. Fonte and V. Peskov, High resolution TOF with RPCs, preprint LIP/00-04, 2000

[11] The ALICE collaboration: Addendum to the technical design report of the time of flight system (TOF), CERN-LHCC-2000-016, ALICE-TDR-8-ADD-1, 2002

[12] GARFIELD, Simulation of gaseous detectors, Version 7.04, 2001, CERN

[13] Discussions at the 'RPC 2001' - 6th Workshop on Resistive Plate Chambers and Related Detectors, 26-27 November 2001, Coimbra, Portugal

[14] W. Riegler, R. Veenhof and C. Lippmann, Detector physics and simulation of resistive plate chambers, CERN-EP-2002-046, Nucl. Instrum. Meth. A 500 (2003) 144-162

[15] M. Abrescia et al., Progress in the simulation of resistive plate chambers in avalanche mode, Nucl. Instr. Meth. B (Proc. Suppl., 1999) 459-464

[16] G. Aielli, Advanced studies on RPCs, Dissertation, Università Degli Studi Di Roma 'Tor Vergata', Rome, Italy, December 2000, 
[17] G. Aielli, Logistic Saturated Avalance Model, presented at the 'RPC 2001' - 6th Workshop on Resistive Plate Chambers and Related Detectors, 26-27 November 2001, Coimbra, Portugal

[18] P. Fonte, High resolution timing of MIPs with RPCs - a model, Nucl. Instr. Meth. A 456 (2000) 6-10

[19] Th. Heubrandtner, B. Schnizer, C. Lippmann and W. Riegler, Static electric fields in an infinite plane condensor with one or three homogeneous layers, CERN-OPEN 2001-074

[20] Th. Heubrandtner, B. Schnizer, C. Lippmann and W. Riegler, Static electric fields in an infinite plane condensor with one or three homogeneous layers, Nucl. Instr. and Meth. 489 (2002), 439-443

[21] C. Lippmann and W. Riegler, Space charge effects and induced signals in resistive plate chambers, presented at the 'RPC 2001' - 6th Workshop on Resistive Plate Chambers and Related Detectors, 26-27 November 2001, Coimbra, Portugal

[22] C. Lippmann and W. Riegler, Detector physics of resistive plate chambers, presented at the Nuclear Science Symposium and Medical Imaging conference, 12-14 November 2002, Norfolk, Virginia, USA

[23] S. Biagi, MAGBOLTZ, Program to compute gas transport parameters, Version 2.2, CERN

[24] S. Biagi, IMONTE, Program to compute gas transport parameters, Version 4.5, CERN

[25] I. Smirnov, HEED, Program to compute energy loss of fast particles in gases, Version 1.01, CERN

[26] Eduardo Gorini, Measurements of drift velocity and amplification coefficient in $\mathrm{C}_{2} \mathrm{H}_{2} \mathrm{~F}_{4}$ Isobutane mixtures for avalanche operated RPC, presented at the 'RPC 1997' - 4th Workshop on Resistive Plate Chambers and Related Detectors, October 1997, Napoli, Italy

[27] V. Golovatyuk, Laser beam studies of RPC behaviour in avalanche mode, presented at the 'RPC 2001' - 6th Workshop on Resistive Plate Chambers and Related Detectors, 26-27 November 2001, Coimbra, Portugal

[28] S. Ramo, Currents induced in electron motion, PROC. IRE 27 (1939) 584

[29] P. Camarri et al., Streamer suppression with $\mathrm{SF}_{6}$ in RPCs operated in avalanche mode, Nucl. Instr. and Meth. A 414 (1998) 317-324

[30] G. Aielli et al., RPC ageing studies, Nucl. Instrum. Meth. A 478 (2002) 271-276

[31] A. Blanco et al., A new front-end electronics chain for timing RPCs, IEEE Trans. Nucl. Science, vol. 48, no. 4 (2001)

[32] P. Fonte, Resistive plate chambers for time of flight, Talk presented at the 'Compressed Baryonic Matter Workshop', May 13-16, 2002, Gesellschat für Schwerionenforschung (GSI), Darmstadt, Germany 